\title{
Operando Analyses of Highly Enhanced Water Splitting by Electronically Modulated Non-noble Metal Carbides
}

\section{Soumyabrata Roy}

Jawaharlal Nehru Centre for Advanced Scientific Research

\section{Debabrata Bagchi}

Jawaharlal Nehru Centre for Advanced Scientific Research

\section{Lakshay Dheer}

Jawaharlal Nehru Centre for Advanced Scientific Research

\section{Saurav Sarma}

Jawaharlal Nehru Centre for Advanced Scientific Research

Vincent Rajaji

Jawaharlal Nehru Centre for Advanced Scientific Research

Chandrabhas Narayana

Jawaharlal Nehru Centre for Advanced Scientific Research

Umesh Waghmare

Jawaharlal Nehru Centre for Advanced Scientific Research https://orcid.org/0000-0002-9378-155X

Sebastian Peter ( $\boldsymbol{\nabla}$ sebastiancp@jncasr.ac.in )

Jawaharlal Nehru Centre for Advanced Scientific Research

\section{Article}

Keywords: Water splitting, alkaline electrolyzer, transition metal carbides, operando XAS analyses

Posted Date: January 6th, 2021

DOl: https://doi.org/10.21203/rs.3.rs-127090/v1

License: (c) (1) This work is licensed under a Creative Commons Attribution 4.0 International License. Read Full License 


\title{
Operando Analyses of Highly Enhanced Water Splitting by Electronically Modulated Non- noble Metal Carbides
}

\author{
Soumyabrata Roy ${ }^{1,2},{ }^{\#}$ Debabrata Bagchi ${ }^{1,2},{ }_{\text {, Lakshay Dheer }}^{2,3}$, Saurav Ch. Sarma ${ }^{1,2}$, Vincent Rajaji ${ }^{2,3}$, Chandrabhas
} Narayana $^{2,3}$, Umesh V. Waghmare ${ }^{2,4}$ and Sebastian C. Peter ${ }^{1,3}$

${ }^{1}$ New Chemistry Unit, Jawaharlal Nehru Centre for Advanced Scientific Research, Bangalore, India ${ }^{2}$ School of Advanced Materials, Jawaharlal Nehru Centre for Advanced Scientific Research, Bangalore 560064 , India ${ }^{3}$ Chemistry and Physics of Materials Unit, Jawaharlal Nehru Centre for Advanced Scientific Research, Bangalore 560 064, India ${ }^{4}$ Theoretical Sciences Unit, Jawaharlal Nehru Centre for Advanced Scientific Research, Bangalore 560 064, India

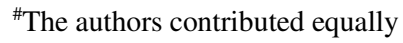

Abstract

Designing non-noble metal based electrocatalysts for efficient overall water splitting (OWS) solves a critical bottleneck of sustainable water electrolyzer technology. Here, we elucidate real-time mechanistic insights into the promotional effect of Ni substitution on the bifunctional OWS activity of N-doped graphite carbon (NGC) supported molybdenum and tungsten carbides (Ni-MoC/WC@NGC). Ni substitution yields multi-fold improvement in water splitting activity over the pristine systems that are comparable to commercial Pt/C for HER and better than $\mathrm{IrO}_{2}$ in case of OER. Ni-MoC@ NGC champions in HER activity exhibiting an onset overpotential of $65 \mathrm{mV}$ and current density of $140 \mathrm{~mA} / \mathrm{cm}^{2}$ at $-370 \mathrm{mV}$ (v RHE) in acidic media. Their excellent OWS activity in alkaline media prompted us to construct a $\mathrm{H}_{2} \mathrm{O}$ electrolyzer where the bifunctional Ni-MoC@ NGC system shows a comparable cell voltage to the $\mathrm{Pt}^{\mathrm{I} I \mathrm{IrO}} 2$ pair and could split water aided by a $1.5 \mathrm{~V}$ AAA commercial battery. First principle calculations and in-situ probing of the local $\mathrm{Ni}$ and $\mathrm{W}$ sites through quick-XAS during electrochemical processes provide valuable insights into how the adsorption energies of intermediates and reaction kinetics are modulated at different catalytic sites with the promotional electronic effect of $\mathrm{Ni}$ substitution.

Keywords: Water splitting; alkaline electrolyzer; transition metal carbides; operando XAS analyses

*Corresponding author. Phone: 080-22082998, Fax: 080-22082627

sebastiancp@jncasr.ac.in (S. C. Peter)

\section{Introduction}

The juxtaposing global scenario of increasing energy demands $\left(1.1 \times 10^{21} \mathrm{~J} / \text { hour by } 2050\right)^{1}$ and incumbent regulations on carbonaceous fossil fuels, triggered by an alarming increase in atmospheric $\mathrm{CO}_{2}$ level $(\sim 410 \mathrm{ppm})$, calls for urgent scientific breakthroughs in establishing alternative fuel economies. ${ }^{2}$ Hydrogen, owing to its high gravimetric energy density (143 kJ kg $\left.{ }^{1}\right)$ and environmentally benign nature, poses as a potential alternative green fuels. ${ }^{3}$ Electrocatalytic water splitting, empowered by renewable energy sources, is one of the most efficient, and sustainable technologies for pure $\mathrm{H}_{2}$ generation that can be easily coupled with fuel cell applications. ${ }^{4-6}$ Thermodynamically uphill processes of hydrogen evolution (HER) at cathode and oxygen evolution (OER) at anode involves overpotentials, which determines the overall cell voltage $\left(E_{\text {cell }}\right)$ governing an electrolyzer's practical viability. ${ }^{7,8}$ In spite of extensive research explorations over the last decade, ${ }^{9-14}$ both acidic and alkaline water electrolysis, alike, are still most efficiently catalyzed by noble metal based catalysts (Pt for HER and Ir/Ru oxides for OER) $)^{4,7,15}$ which are prohibitively expensive and scarce. ${ }^{16,17}$ Thus, new catalyst engineering concepts influencing and progressing the limits of the state-of-the-art is of utmost importance in this regard.

Earth-abundant transition metal (TM) based electrocatalysts (phosphides, ${ }^{18-21}$ carbides, ${ }^{10,22-24}$ nitrides, ${ }^{25-27}$ borides, ${ }^{12,28}$ chalcogenides ${ }^{29-35}$ ) have strongly emerged as a new class of lowcost and efficient electrocatalysts for HER, OER and water splitting. ${ }^{14,31,32,36,37}$ Among them Mo and W carbides (interstitial compounds $)^{38}$ stands out owing to their noble metal like electronic structure around Fermi level, ${ }^{39}$ high electrochemical stability and adaptability to different structural engineering techniques. ${ }^{3,6,40} \mathrm{~A}$ plethora of interesting approaches ${ }^{41-43}$ has been undertaken over the last few years to improve the activities of pristine Mo and W carbide which include phase, shape \& size modulation, $, 3,444-47$ active site enhancement, interface/strain engineering,, $548-50$ introducing interacting supports, ${ }^{6,51,52}$ incorporating heteroatoms like $\mathrm{N}^{40,53}$ alloying or doping secondary metals ${ }^{43,54}$ etc. In spite of these efforts, till date, very few reports exist for structurally engineered Mo and W carbides exhibiting highly efficient overall water splitting, with equivalent cathodic and anodic activity. $.5,12,40$

To achieve the desired activities it is important to understand the underlying catalytic features governing the descriptors that modulate the electrochemical processes. Optimizing adsorbatemetal interactions, d-band centre tuning, increasing ECSA and chemical state modulation of active centres are among the most important factors that can modulate a catalyst's electrochemical activity. ${ }^{32,48,55-62}$ ECSA and partial oxidation states of active metals, on the other hand, determines the site-density and electron transfer kinetics of an electrocatalyst. To tap into the potential of all these factors, tuning chemical composition and electronic structures of W and Mo centres by substitution of a secondary TM appears as one of the most efficient and facile approaches..$^{14,17,54,60}$ In this work, we have chosen $\mathrm{Ni}$ as the suitable substituent owing to its superior activity for HER/OER in both acidic and alkaline media, valence electron rich surface and adaptability to charge transfer interactions. ${ }^{5,60,63-66}$ The proton adsorption capability and high electrical conductivity of defect enriched $\mathrm{N}$-doped graphitic carbon (NGC) ${ }^{6,67,68}$ prompted us to exploit its potential as an useful support for dispersing and stabilizing the $\mathrm{Ni}$-doped $\mathrm{Mo}$ and $\mathrm{W}$ carbide nanoparticles (NPs).

Through a one-step in-situ carbonization technique we synthesized Ni substituted WC and MoC nano-systems dispersed on NGC. Both the catalysts (Ni-WC@NGC \& Ni-MoC@NGC) exhibit multi-fold improvement in activity in terms of current densities, onset values, Tafel slopes and electron transfer resistances as compared to the undoped carbide catalysts. Synthetic optimization yields Ni-MoC(1:5)@NGC as the best HER electrocatalysts in acidic media with an onset overpotential of 65 $\mathrm{mV}$ and current density of $140 \mathrm{~mA} / \mathrm{cm}^{2}$ at $-370 \mathrm{mV}$ (v RHE). Both Mo and $\mathrm{W}$ samples show enhanced and durable OER activity in alkaline medium with onsets of $1.5 \mathrm{~V}$ (vs. RHE) and current densities as high as $150 \mathrm{~mA} / \mathrm{cm}^{2}$ at $1.86 \mathrm{~V}$, which is close to stateof-the-art activity as bifunctional OWS catalysts triggered us to construct a low $\mathrm{E}_{\text {cell }} \mathrm{H}_{2} \mathrm{O}$ electrolyzer device. Ni-MoC@NGC exhibits a comparable cell voltage to the $\mathrm{Pt}^{\mathrm{IIO}} \mathrm{I}_{2}$ pair and can split water aided by a $1.5 \mathrm{~V}$ AAA commercial battery. The electronic and structural roles of $\mathrm{Ni}$ in enhancing the catalytic activity have been extensively studied through operando quick X-ray absorption spectroscopic (QXAS) studies and density functional theory (DFT) calculations. In-situ probing of the local $\mathrm{Ni}$ and $\mathrm{Mo} / \mathrm{W}$ sites during different electrochemical processes provides valuable insights into relative mechanisms of HER and OER in different media. These studies elucidate, in real time picture, how the adsorption energies of intermediates and reaction kinetics are modulated at different catalytic sites with the promotional effect of $\mathrm{Ni}$ substitution.

\section{Results and Discussions}

\subsection{Synthesis, Structural Analyses and Characterization}

The Ni-substituted W/Mo carbide-based hybrid nanocatalysts are synthesized through a facile one-step synthetic procedure following the scheme shown in Figure 1a. The precursors, polyoxometalate $\left(\mathrm{H}_{3} \mathrm{PMo}_{12} \mathrm{O}_{40} / \mathrm{H}_{3} \mathrm{PW}_{12} \mathrm{O}_{40}\right)$, nickel chloride $\left(\mathrm{NiCl}_{2}\right)$, and melamine $\left(\mathrm{C}_{3} \mathrm{H}_{6} \mathrm{~N}_{6}\right)$ are thoroughly mixed at various molar ratios of Mo/W:Ni $(5: 1,5: 2,5: 3)$ followed by subsequent ball milling. The ball-milled mixture is vacuum-sealed in quartz tube and pyrolyzed at $900{ }^{\circ} \mathrm{C}$ (based on the TGA, Figure S1, and suitable temperature profile, Figure S2) resulting in the formation of well-dispersed Ni-substituted Mo and $\mathrm{W}$ carbide NPs on nitrogen-doped graphitic carbon (Ni-W/MoC@NGC, see synthetic details in ESI for details, Figure 1b-d, S3-S4, Figure 2). 
(a)
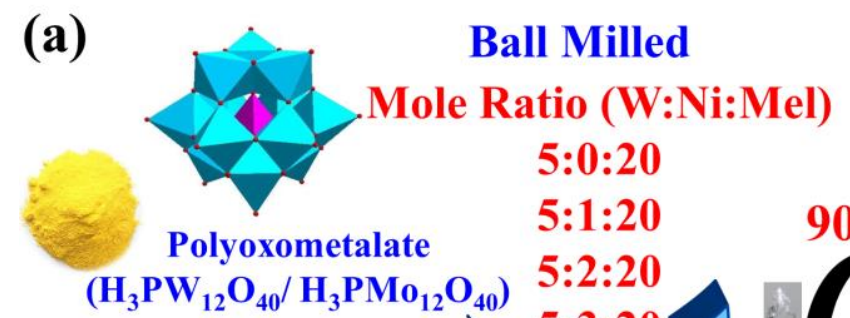

$5: 1: 20$
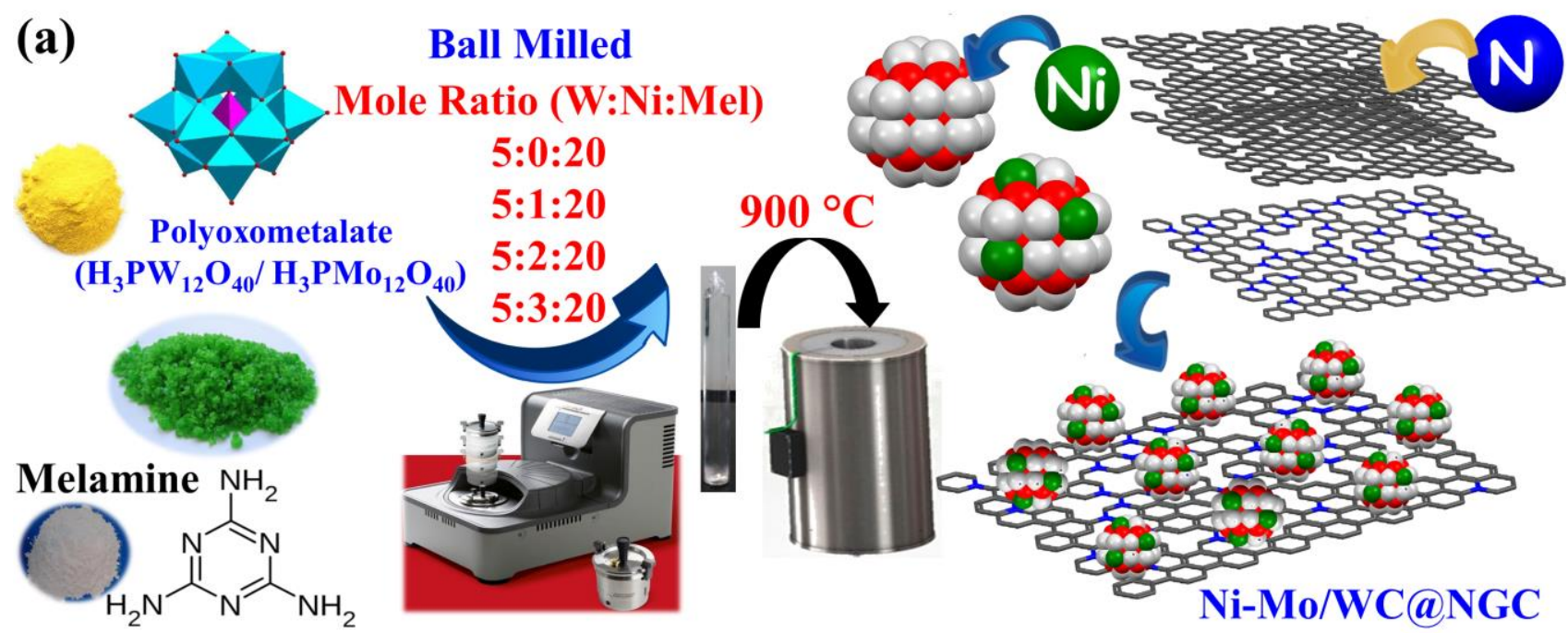

(b)

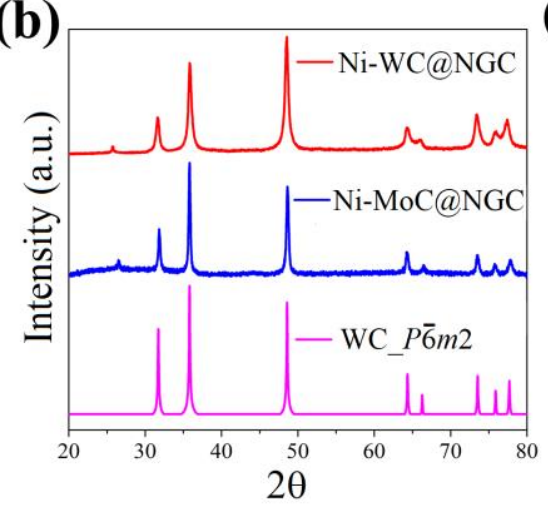

(c)

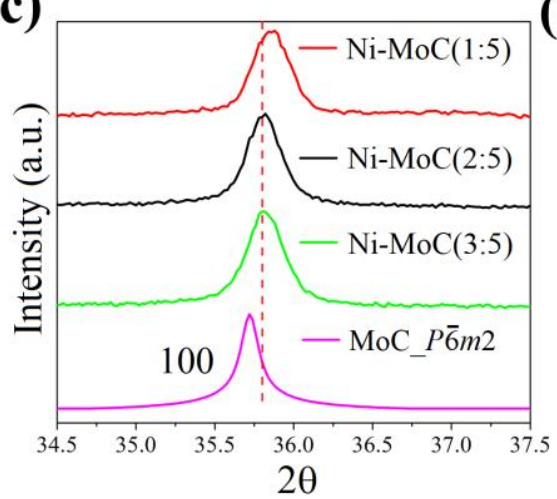

(d)

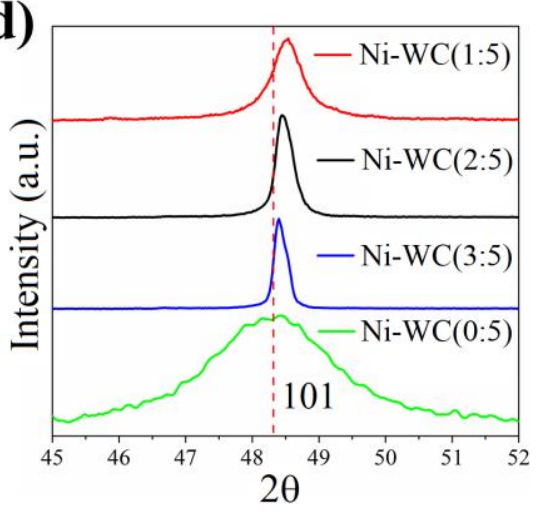

Figure 1. Synthesis and characterization of Ni-WC@NGC \& Ni-MoC@NGC: (a) Synthetic scheme for Ni-W/MoC@NGC showing different steps of precursor mixing, carbonization and $\mathrm{Ni}$ substituion into W/MoC lattice and $\mathrm{N}$ doping in carbon matrix, (b) Comparative PXRD patterns of Ni-WC@NGC \& Ni-MoC@NGC, (c) PXRD shift in 100 plane of Ni-MoC having different precursor mole ratios and (d) PXRD shift in 101 plane of Ni-MWC having different precursor mole ratios.

In addition to enabling efficient diffusion of $\mathrm{Ni}$ into the metal carbide lattice, ball milling also enhances the microstructural disordering and defects in the graphitic carbon, ${ }^{5}$ as evident from the comparative $\mathrm{I}_{\mathrm{D}} / \mathrm{I}_{\mathrm{G}}$ ratios (Figure S4-S5). Melamine precursor acts as the reductant, which carbonised the metal-oxy clusters, onto a graphitic carbon matrix, enriched in nitrogen from the melamine molecular unit. Gases like $\mathrm{CH}_{4}, \mathrm{NH}_{3}$ generated in-situ from melamine at high temperatures induce a chemical pressure facilitating Ni diffusion and dopes the carbon sheets with nitrogen during graphitisation. The nitrogen defects enhances the electronic properties and conductivity of the graphitic carbon promoting efficient proton adsorption during electrocatalysis. ${ }^{53,68,69}$ The same precursor mixture calcined under $\mathrm{H}_{2} / \mathrm{Ar}$ in an open boat, results in additional metallic oxide impurities owing to incomplete carbonization \& Ni diffusion. Thus, the use of vacuum-sealed quartz ampules is essential to ensure an oxygen free atmosphere and induction of chemical vapour pressure for $\mathrm{Ni}$ diffusion and $\mathrm{N}$ doping. The substituted carbides crystallize in the hexagonal $P \overline{6} \mathrm{~m} 2$ space group of MoC/WC phase (Figure 1b) with right shift in the diffraction peak positions (Figure 1c-(100) of $\mathrm{MoC}$ at $35.7^{\circ}$, 1d(101) of $\mathrm{WC}$ at $48.4^{\circ}$ ) due to lattice shrinkages on substitution of the smaller $\mathrm{Ni}$ ions $\left(\mathrm{Ni}^{2+}: 69 \mathrm{pm}, \mathrm{Mo}^{6+}: 79 \mathrm{pm}\right.$ and $\left.\mathrm{W}^{6+}: 80 \mathrm{pm}\right)$ in the carbide lattices. ${ }^{70}$ The absence of additional peaks of metallic $\mathrm{Ni}$ or nickel carbide phases implies the substitution of $\mathrm{Ni}$ in the carbide lattice. Molar ratio of 5:1 with respect to $\mathrm{W}: \mathrm{Ni}$ is found to be the optimum, as higher $\mathrm{Ni}$ percentages $(\mathrm{Ni}-\mathrm{WC}(2: 5)$ or NiWC(3:5)) lead to similar or lesser Ni substitution with excess of metallic Ni phases (Figure S3a, peaks at $44.3^{\circ} \& 51.6^{\circ}$ ), owing to solid solubility limit. The controlled reactions, devoid of $\mathrm{Ni}(\mathrm{Ni}-$ $\mathrm{WC}(0: 5)$ or Ni-MoC(0:5)), yield mixed phases of $\mathrm{MoC}(F m \overline{3} m)$ and $\mathrm{Mo}_{2} \mathrm{C}$ (Pbcn), (Figure S3b) for Mo, while primary phase of WC $(P \overline{6} \mathrm{~m} 2)$ was obtained in the case of $\mathrm{W}$ variant. Ni incorporation triggered the formation of the hexagonal phase in both Mo and $\mathrm{W}$ systems, through substantial lowering of the formation energy (values, Table S1). Interestingly, profile fitting (Figure S3c, d), revealed that Ni substitution affected the relative intensities of the (100) (MoC-Structure Factor (SF):31, WC-SF:54) and (101) (MoC-SF:29, WC-SF:50) plane differently in case of Mo and W carbide systems (see ESI for details). In case of MoC, the intensity of (100) plane (consisting of only Mo) at $35.7^{\circ}$ was enhanced, while in WC the (101) (having structure factors from both $\mathrm{W}$ and $\mathrm{C}$ atoms) at $48.4^{\circ}$ plane reached a higher intensity than the (100) plane (Figure S3e, $\mathbf{f}$ )

Elemental analyses of Ni-Mo/WC@NGC ( $\left.\mathrm{X}_{5} \mathrm{Ni}_{1} \mathrm{C} @ \mathrm{NGC}\right)$ was carried out through FESEM-EDS, TEM-EDS and ICP studies (Table S2, S3). From a cumulative elemental analyses, it can be seen that ball milling (BM) enhances the $\mathrm{Ni}$ diffusion into the carbide lattice. The average ratio of Ni:Mo/W (1:4.16 for Mo, 1:4.27 for $\mathrm{W}$ ) for the ball-milled sample was double of that (Ni:Mo/W = 1:9.19 Mo, 1:8.77 W) in the non-ball milled samples (Table S3). TEM images show that the Ni-substituted W/MoC (particle size $\sim 10-30 \mathrm{~nm}$ ) nanoparticles are encapsulated and welldispersed over the graphitic carbon matrix (Figure 2). The incorporation of $\mathrm{Ni}$ in the $\mathrm{Mo} / \mathrm{W}$ carbide lattice is further confirmed from the elemental mapping on selected particles which showed a uniform mixed distribution of $\mathrm{Ni}$ and $\mathrm{Mo} / \mathrm{W}$ (Figure 2a, b and $2 \mathbf{j}, \mathbf{k}$ ). High resolution TEM (HRTEM) images, show a dspacing of $0.249 \mathrm{~nm}$ corresponding to the 100 plane (Figure $2 \mathbf{h}$ ) in the Ni-MoC system and $0.28 \& 0.25 \mathrm{~nm}$ for the Ni-WC corresponding to 001 and 100 facets (Figure $\mathbf{2 m}$ ), respectively. Selected area electron diffraction (SAED) patterns display the concentric rings of diffractions, characteristic of the low index exposed planes $(001,100,101)$ in either systems (Figure 2d, 2n). Elemental line scanning on the particles of Ni-MoC@NGC (Figure 2g) confirms the Ni substitution in the carbide structure. SEM images show the microstructural arrangement where the semi-spherical carbide NPs are found to be grafted on the thicker carbon matrix (Figure S4a, S4b). 

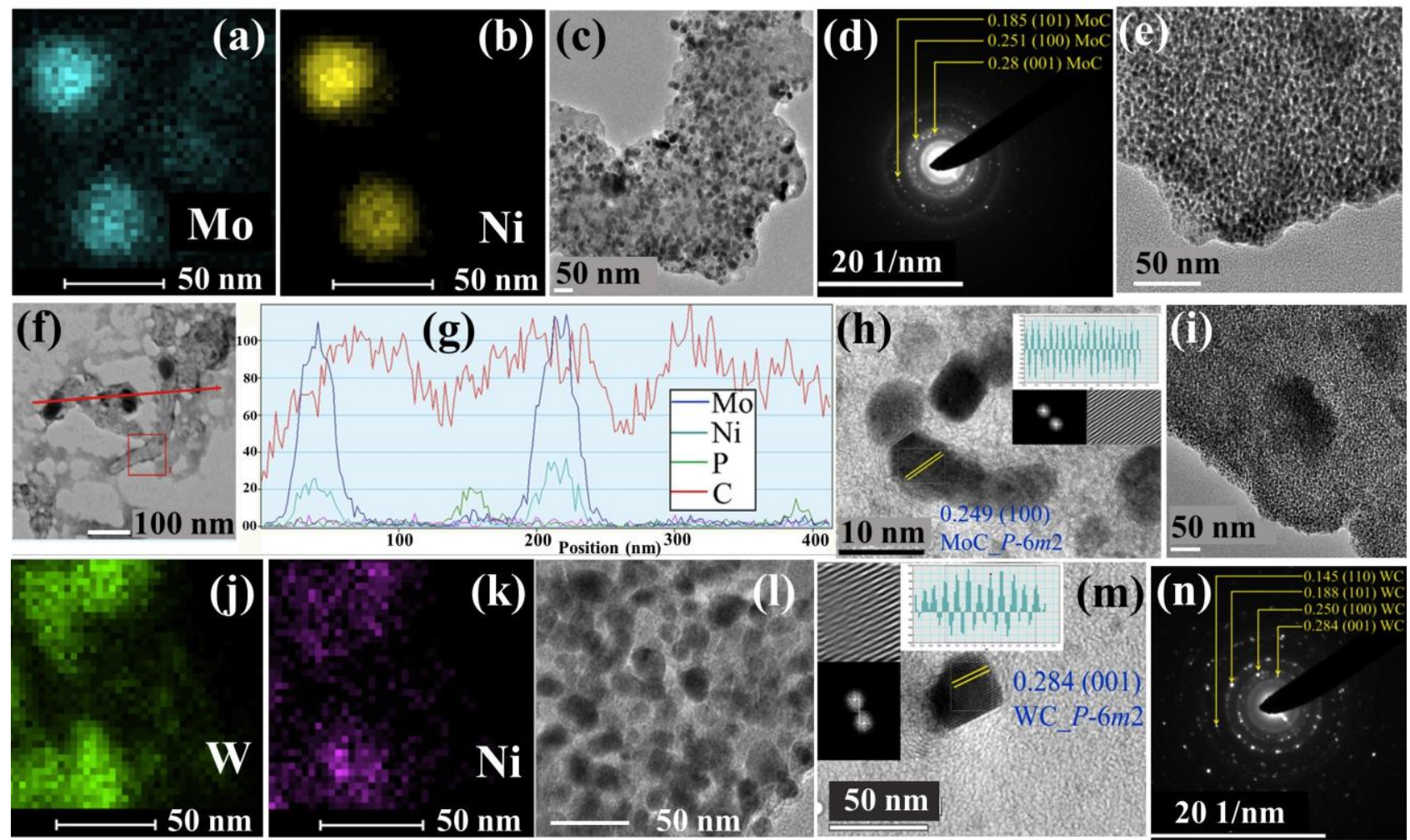

Figure 2. TEM analysis of Ni-WC@NGC \& Ni-MoC@NGC: (a) \& (b) Colour mapping on particles of Ni-MoC@ NGC, (c) \& (e) Particle distribution in Ni-MoC@NGC, (d) SAED pattern of Ni-MoC@NGC showing presence of corresponding lattice planes, (f) \& (g) Line scanning EDS spectra of Ni-MoC@NGC, (h) HR-TEM image Ni-MoC@NGC particle showng the d-spacing corresponding to 100 plane, (i) \& (l) Particle distribution in Ni-WC@NGC, (j) \& (k) Colour mapping on particles of Ni-WC@NGC, (m) HR-TEM image NiWC@NGC particle showng the d-spacing corresponding to 001 plane and (n) SAED pattern of Ni-WC@NGC showing presence of corresponding lattice planes.

XPS studies (Figure S4c, S4d) depicts the electronic effect of $\mathrm{Ni}$ substitution on $\mathrm{Mo} / \mathrm{W}$, where in addition to the expected $\mathrm{Mo} / \mathrm{W}^{+4}$ peaks, a notable presence of $\mathrm{Mo}$ and $\mathrm{W}^{+6}$ species can be seen. This is attributed to the substitution of $\mathrm{Ni}$, which being a divalent cation induces an increase in the usual oxidation state of $\mathrm{Mo} / \mathrm{W}$ ( +4 in carbides) to +6 in the substituted system. Near surface composition analyses through XPS also prove the elemental presence and electronic nature of $\mathrm{C}$, and $\mathrm{N}$ in the nano hybrids (Figure S5a). The peaks centred at 284.6, 285.8, 287, $289.7 \mathrm{eV}$ of the C1s spectra (Figure S5b) correspond to C-C/ $\mathrm{C}=\mathrm{C}, \mathrm{C}-\mathrm{N}, \mathrm{C}-\mathrm{O}$ and $\mathrm{C}=\mathrm{O}$ of the graphitic carbon sheets. The typical presence of pyrrolic $(397.5 \mathrm{eV})$, pyridinic (394.8) and graphitic $(401.5 \mathrm{eV}) \mathrm{N}$ species in the $\mathrm{N}$ 1s spectra (Figure S5c) confirm the doping of $\mathrm{N}$ in the carbon matrix. ${ }^{71,72}$ Furthermore, Raman studies manifest the degree of graphitisation in the carbonized nanocomposites with enhanced $\mathrm{D}$ and $\mathrm{G}$ bands around 1350 and $1598 \mathrm{~cm}^{-1}$ respectively. The $\mathrm{I}_{\mathrm{D}} / \mathrm{I}_{\mathrm{G}}$ values obtained (Figure S6a, b, see the SI for details) indicate the presence of defects, attributable to $\mathrm{N}$ doping in the carbon matrix. This is expected to favours active site accessibility, electrical conductivity and enhanced electrochemical performance in the composites. ${ }^{3,6,40}$ 2.2. Hydrogen Evolution Reaction (HER)

The series of Ni-W/MoC@NGC compounds were investigated for electrochemical HER activity in a conventional three-electrode setup under acidic and basic conditions. Figure 3a shows a comparison of the polarization curves of W/MoC@NGC, NiW/MoC@NGC, $20 \mathrm{wt} \% \mathrm{Pd} / \mathrm{C}$ and $20 \mathrm{wt} \% \mathrm{Pt} / \mathrm{C}$ obtained from the iR-corrected linear sweep voltammograms at a scan rate of 5 $\mathrm{mV} / \mathrm{sec}$. Multi-fold enhancement in terms of onset potential and current densities can clearly be observed, in the Ni-substituted samples as compared to the undoped carbides. Though Ni samples exhibit similar onset potentials (at $66 \mathrm{mV}$ for Ni-WC@NGC and 69 mV of Ni-MoC@NGC), Ni-MoC@NGC excels in the overall activity, in terms of current density and the reaction kinetics (Figure 3a, Table S4).

The marked decrease in the Tafel slope values (Figure S7a, Table S4) from the undoped carbides to the Ni-substituted ones indicates a clear enhancement in reaction kinetics and mechanism, stemming from a more facile electron and mass transfer process in the modulated catalysts. The Tafel slope values which is an indicative of the mechanism and the rate determining step (r.d.s.) of HER, show that the Ni-substituted carbides exhibit intermediate Tafel values (between Volmer and Heyrovsky) signifying occurrence of spill over mechanism. ${ }^{17}$ Both the Ni-substituted catalysts show highly enhanced ( $\sim 10$ times higher) intrinsic exchange current densities (jo) of $0.32 \times 10^{-4} \mathrm{~A} / \mathrm{cm}^{2}$ (NiWC@NGC) and $0.56 \times 10^{-4} \mathrm{~A} / \mathrm{cm}^{2}$ as compared to the unsubstituted systems (WC@NGC: 0.34 x $10^{-5} \quad \mathrm{~A} / \mathrm{cm}^{2} \quad \&$ MoC@NGC: $\left.0.79 \times 10^{-5} \mathrm{~A} / \mathrm{cm}^{2}\right) .^{15,17}$ The Nyquist plots obtained from electrochemical impedance spectroscopy (EIS) reveal that the $\mathrm{Ni}$-substituted nano-carbides suffer extremely low charge-transfer resistance $\left(\mathrm{R}_{\mathrm{CT}}\right.$, one order less: $117 \Omega$ for Ni-WC@NGC \& $78 \Omega$ for Ni-MoC@NGC) (Figure 3b), as compared to the undoped catalysts (1169 for WC@NGC \& 530 for MoC@NGC). The decrease in charge-transfer resistance signifies enhancement in electron transfer kinetics during electrocatalysis, arising from $\mathrm{Ni}$ substitution. Ni-MoC@NGC which exhibits quite low Tafel slope value $(76 \mathrm{mV} / \mathrm{dec})$ and $\mathrm{R}_{\mathrm{CT}}(78 \Omega)$ for HER in acidic media is seen to approach the activity of the state-of-the-art catalysts $\mathrm{Pd} / \mathrm{C}$ and $\mathrm{Pt} / \mathrm{C}$ at higher potentials (Figure 3a). Between the unsubstituted and Ni-substituted MoC@NGC catalysts we observe a decrease of $\sim 150 \mathrm{mV}$ in onset, $\sim 5$ fold increase in current density (@ -370 $\mathrm{mV}), \sim 30 \mathrm{mV} / \mathrm{dec}$ decrease in Tafel slope and almost an order decrease in the charge transfer resistance (@-0.13 V). Thus, all in all it can be concluded that the Ni-substituted carbide catalysts exhibit supreme catalytic activity surpassing most of the earlier reported carbides and non-noble metal catalysts tested under similar conditions (Table S5).

The catalysts are ultrastable under both electrochemical cycling (accelerated durability test, ADT, Figure S7b) and chronoamperometric modes of stability tests (@10 mA/cm (Figure S7c, d). A marginal increase in activity during the initial 250 cycles (ADT between 0 to $-0.33 \mathrm{~V}$ vs RHE) (Figure S7b), can be attributed to the leaching induced vacant sites creation, and chemical state optimization of the active centers. ${ }^{73}$ However, after the marginal dissolution of $\mathrm{Ni}$ and $\mathrm{W} / \mathrm{Mo}$ atom during the initial cycles, the catalysts (Ni-WC@NGC, Ni-MoC@NGC) show high electrochemical durability till 2000 cycles with no further change in the polarization current. 
(a)

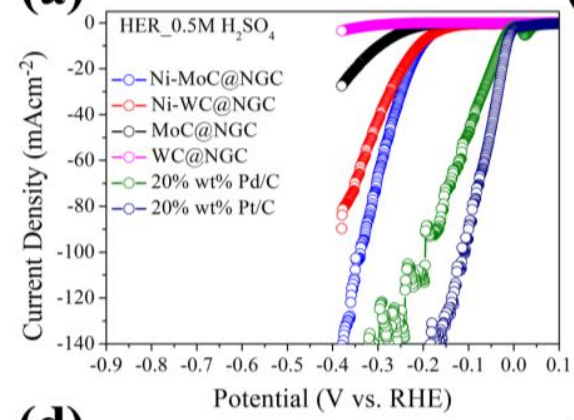

(d) Potential (V vs, RHE)

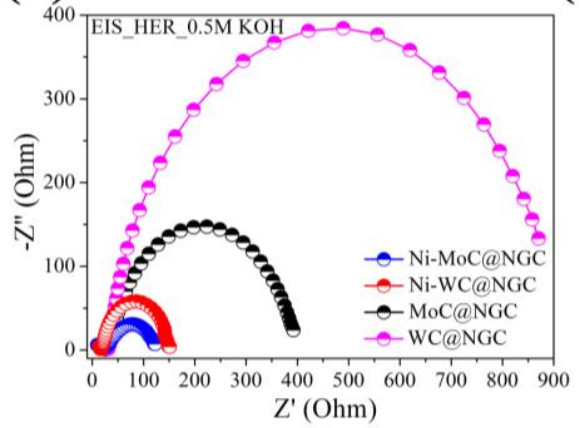

(b)

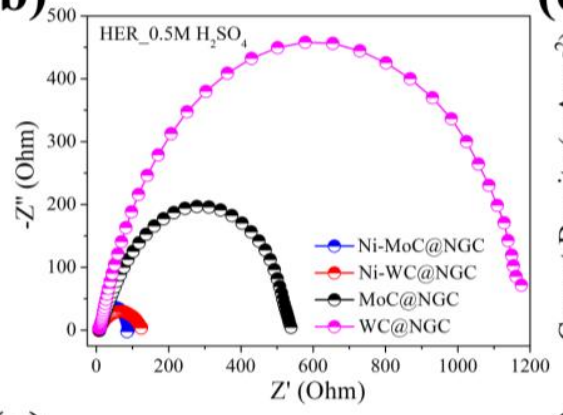

(e)

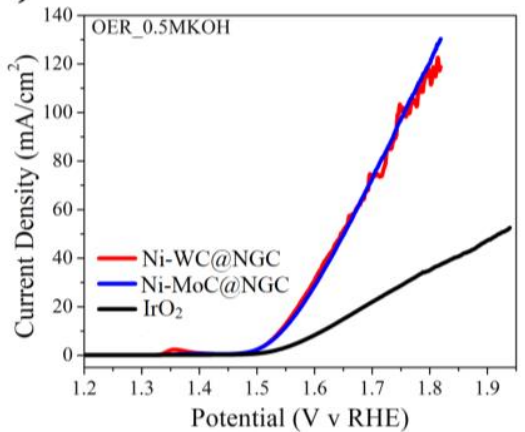

(c)

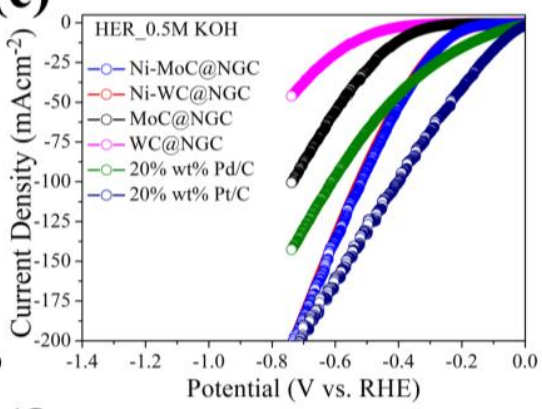

(f)

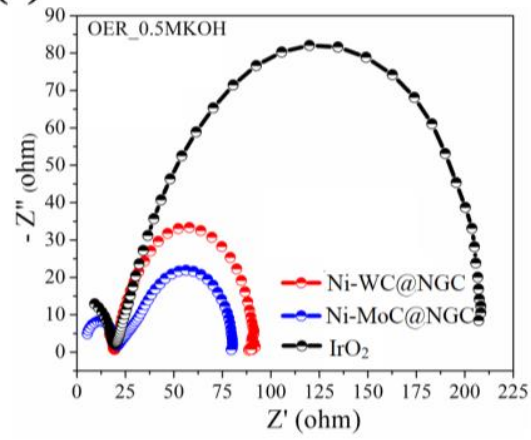

Figure 3. (a) Comparative polarization curves of Ni-WC@NGC \& Ni-MoC@NGC for HER in $0.5 \mathrm{M} \mathrm{H}_{2} \mathrm{SO}_{4}$ under a scan rate of $5 \mathrm{mV} / \mathrm{s}$ using SCE as the reference and Pt coil as the counter electrode. (b) Electrochemical Impedence Spetra (EIS) of Ni-WC@NGC, NiMoC@NGC and undoped catalysts showing R R during HER in $0.5 \mathrm{M} \mathrm{H}_{2} \mathrm{SO}_{4}$. (c) Comparative polarization curves of Ni-WC@ NGC \& NiMoC@NGC with Pd/C \& Pt/C for HER in 0.5M KOH. (d) EIS spectra of Ni-WC@NGC, Ni-MoC@NGC and undoped catalysts showing $\mathrm{R}_{\mathrm{CT}}$ during HER in $0.5 \mathrm{M} \mathrm{KOH}$. (e) Comparative polarization curves of Ni-WC@NGC, Ni-MoC@NGC and $\mathbf{I r O}_{2}$ for electrochemical OER in $0.5 \mathrm{M} \mathrm{KOH}$ under a scan rate of $5 \mathrm{mV} / \mathrm{s}$ using $\mathrm{Hg} / \mathrm{HgO}$ as the reference and Pt coil as the counter electrode. (f) EIS spectra of NiWC@ NGC, Ni-MoC@ NGC and $\mathbf{I r O}_{2}$ showing R $\mathrm{R}_{\mathrm{CT}}$ during OER in $0.5 \mathrm{M} \mathrm{KOH}$.

The catalysts after electrochemical HER were analysed through XANES, XAFS, TEM-imaging and TEM-EDS studies. The slight decrease in the XANES edge jump at Ni before and after HER (250 cycles) (Figure S7e), indicates a slight decrease in $\mathrm{Ni}$ amount as a result of initial electrochemical leaching and activity enhancement. The post-electrochemical comparative elemental and microscopic analyses through TEM (Figure S8) show that the catalysts are structurally and compositionally stable. Electrochemically active surface area (ECSA) is a critical kinetic factor influencing the activity and current densities of a catalyst. ${ }^{15}$ To deconvolute the role of ECSA in activity enhancement, double layer capacitances $\left(\mathrm{C}_{\mathrm{dl}}\right)$ of the catalysts are determined (Figure S7f), where the current response is majorly associated with the double-layer charging (Figure S9) ${ }^{13}$ As shown in Figure S7f, the Ni-substituted catalysts (Ni-WC@NGC: $14.9 \mathrm{mF} / \mathrm{cm}^{2}$, NiMoC@NGC: $21.4 \mathrm{mF} / \mathrm{cm}^{2}$ ) exhibit larger $\mathrm{C}_{\mathrm{dl}}$ values than the pristine carbides (WC@NGC: $3 \mathrm{mF} / \mathrm{cm}^{2}$, MoC@NGC: 10 $\mathrm{mF} / \mathrm{cm}^{2}$ ) which indicates an increase in ECSA upon Nisubstitution. The activity difference between the Ni-WC@NGC and Ni-MoC@NGC catalysts can be partially attributed to the larger ECSA in case of the latter.

As mentioned earlier, the difference in relative intensities and shifts of the (100) and (101) peaks in PXRD (Figure 1b-d, Sc-d) indicates that $\mathrm{Ni}$ is majorly substituting at two different planes in case of the WC and MoC systems. This is further corroborated from the formation energy calculations showing the 100 plane (more exposed than 101) in case of $\mathrm{MoC}$ systems to achieve a higher stabilization energy (-3999 Ha, Table S1) upon $\mathrm{Ni}$ substitution. Mo ionic radii being slightly lesser than that of $\mathrm{W}$ (Lanthanide contraction), may prefer higher d-spacing plane (100) for $\mathrm{Ni}^{2+}$ incorporation.

The effect of synthetic conditions on the electrocatalytic activity is analyzed by testing a number of controlled catalysts like Ni-W/MoC(2:5)@NGC, Ni-W/MoC(3:5)@NGC, NiW/MoC(1:5)-NBM@NGC (NBM: non-ball milled). The polarization curves shown in Figure S10a, b demonstrate that 5:1 W/Mo:Ni ratio yields the best electrochemical activity among the variants of Ni substitution. From diffraction pattern analyses, it can be concluded that the highest $\mathrm{Ni}$ substitution occurs in the case of 5:1 (W/Mo:Ni) sample as higher percentages $(5: 2$ or $5: 3)$ result in elemental $\mathrm{Ni}$ impurities (Figure S3a). Presence of excess elemental $\mathrm{Ni}$ in hetero-structured form or isolated sites does not really enhance the activity. Milling of the precursors is crucial for more $\mathrm{Ni}$ diffusion into the carbide systems, as reflected in the enhancement of HER activity in Ni-W/MoC(1:5)@NGC as compared to the NBM variant (Figure S10c, d). The electrocatalytic studies on the controlled catalysts revealed three important points: (a) Electronic interaction between Ni \& W/Mo centres is the key governing factor for the HER activity, (b) Isolated/electronically non-interacting $\mathrm{Ni}$ cannot enhance the activity to a similar extent and (c) HER activity is proportional to the amount of substituted $\mathrm{Ni}$ in the carbide lattice.

Subsequently, we investigate the HER activity of the Nisubstituted catalysts in alkaline media of $0.5 \mathrm{M} \mathrm{KOH}$. HER activity across extreme $\mathrm{pH}$ ranges from $0.5 \mathrm{M} \mathrm{H}_{2} \mathrm{SO}_{4}$ to $0.5 \mathrm{M}$ $\mathrm{KOH}$ is crucial for practical applications, which is rarely exhibited by non-noble metal electrocatalysts. Both Ni-WC@NGC and NiMoC@NGC catalysts exhibit excellent and equivalent alkaline HER activities with respective low overpotentials (Figure 3c, Table S5). The catalysts exhibit very low Tafel slope values of 135 mV/dec (Ni-WC@NGC) and 124 mV/dec (Ni-MoC@NGC) (Figure S11a, Table S4) as compared to the reported carbide and non-noble metal based systems (Table S5), indicative of facile reaction kinetics. The current densities $\left(200 \mathrm{~mA} / \mathrm{cm}^{2}\right)$ at higher potentials match the activity of state-of-the-art catalyst, $20 \mathrm{wt} \%$ $\mathrm{Pt} / \mathrm{C}$, and surpass $20 \mathrm{wt} \%$ of $\mathrm{Pd} / \mathrm{C}$ at $\sim 60 \mathrm{~mA} / \mathrm{cm}^{2}$. The catalysts exhibit lower Tafel slope values than that of $\mathrm{Pd} / \mathrm{C}(193 \mathrm{mV} / \mathrm{dec})$ in the alkaline media. Multi-fold enhancement in activity in terms of onset potential (Ni-WC@NGC: 120 mV, Ni-MoC@NGC: 90 mV), Tafel slopes and current densities is observed upon $\mathrm{Ni}$ substitution (Table S4). The Ni-substituted catalysts exhibit very low RCT values (Figure 3d, Ni-WC@NGC: $133 \Omega$ \& Ni-MoC@NGC: 95 $\Omega$ ) indicative of facile electron transfer processes during the electrocatalysis. HER activity of most non-Pt catalysts in alkaline media suffers from the sluggish water activation step (usually the r.d.s.) resulting in a more complicated proton adsorption process (Volmer reaction, $\mathrm{H}_{2} \mathrm{O}+\mathrm{e}^{-} \rightarrow \mathrm{H}^{*}+\mathrm{OH}^{-}$, ). ${ }^{13,70,74}$ In case of the pristine W/MoC@NGC for HER in KOH we observe a Tafel slope of $206 \mathrm{mV} / \mathrm{dec}$ and $159 \mathrm{mV} / \mathrm{dec}$ which confirms that the Volmer step (proton adsorption step) is the r.d.s in these carbide catalysts. ${ }^{66,75,76}$ However, a stark decrease in the Volmer limited Tafel slopes in the substituted catalysts indicates that the water activation step becomes more facile upon $\mathrm{Ni}$ substitution. $\mathrm{H}_{2} \mathrm{O}$ activation ( $\mathrm{H}-\mathrm{OH}$ bond breaking) in alkaline condition involves adsorption of the $\mathrm{H}_{2} \mathrm{O}$ molecule on the active site through the interaction of the $\mathrm{O}$ atom with the $\delta(+\mathrm{ve})$ metal ion (in this case 
W/Mo) and that of $\mathrm{H}$ with the $\delta(-\mathrm{ve}) \mathrm{C} .{ }^{17}$ The breaking of the $\mathrm{H}$ $\mathrm{OH}$ bond is facilitated by the induction of a higher partial charge on the W/Mo centres by $\mathrm{Ni}^{2+}$ resulting in an improved activity in the substituted nano-catalysts.

The catalysts exhibit highly durable alkaline HER activity under both dynamic potential (5000 cycles ADT, Figure S11b) with $\leq 1 \%$ degradation in catalytic current densities (after 5000 cycles) and static amperometric conditions ( $24 \mathrm{hrs}, \mathrm{CA})$ with no significant drop at the operating current density of $20 \mathrm{~mA} / \mathrm{cm}^{2}$ for $24 \mathrm{hrs}$ (Figure S11c, d). The activity and stability of Ni-doped W/MoC@NGC (Figure S12a, b) is far better than undoped catalysts (Figure S12c, d) after comparing the LSVs of various catalysts at different number of ADT cycles. The Ni-doped catalysts, unlike in the acidic media, do not show any significant improvement in the polarization curves during initial cycling (S12e), as negligible dissolution of $\mathrm{Ni}$ is observed in the alkaline electrolyte, from the edge jump in Ni XANES spectra before and after electrochemistry (Figure S12f). The similarity in $\mathrm{Ni}$ WC@NGC and Ni-MoC@NGC catalysts' activity in alkaline media can be attributed to the more active role of 101 plane, for HER in $\mathrm{KOH}$ owing to its uniform distribution of metal and carbon atoms.

\subsection{Oxygen Evolution Reaction (OER)}

Figure 3e depicts the OER polarization curves of the substituted catalysts in $0.5 \mathrm{M} \mathrm{KOH}$ in comparison to the benchmark catalyst $\mathrm{IrO}_{2}$. Both Ni-WC@NGC and Ni-MoC@NGC exhibit excellent activities for electrochemical OER with onset potentials of $1.45 \mathrm{~V}$ (vs. RHE) and overpotentials of $310 \mathrm{mV}, 340$ $\mathrm{mV}, 410 \mathrm{mV}$ and $520 \mathrm{mV}$ at $10,20,50$ and $100 \mathrm{~mA} / \mathrm{cm}^{2}$ respectively. The overpotentials obtained for $\mathrm{IrO}_{2}$ under similar conditions are $380 \mathrm{mV}\left(\eta_{10}\right), 460 \mathrm{mV}\left(\eta_{20}\right)$ and $690 \mathrm{mV}\left(\eta_{50}\right)$. The Tafel slopes of Ni-WC@NGC \& Ni-MoC@NGC are $57 \mathrm{mV} / \mathrm{dec}$ \& $60 \mathrm{mV} / \mathrm{dec}$ respectively, which are substantially less than that of $\mathrm{IrO}_{2}(75 \mathrm{mV} / \mathrm{dec})$ (Figure S13a). The $\mathrm{R}_{\mathrm{CT}}$ values (Ni-WC: $71 \Omega$,

\section{(a)}
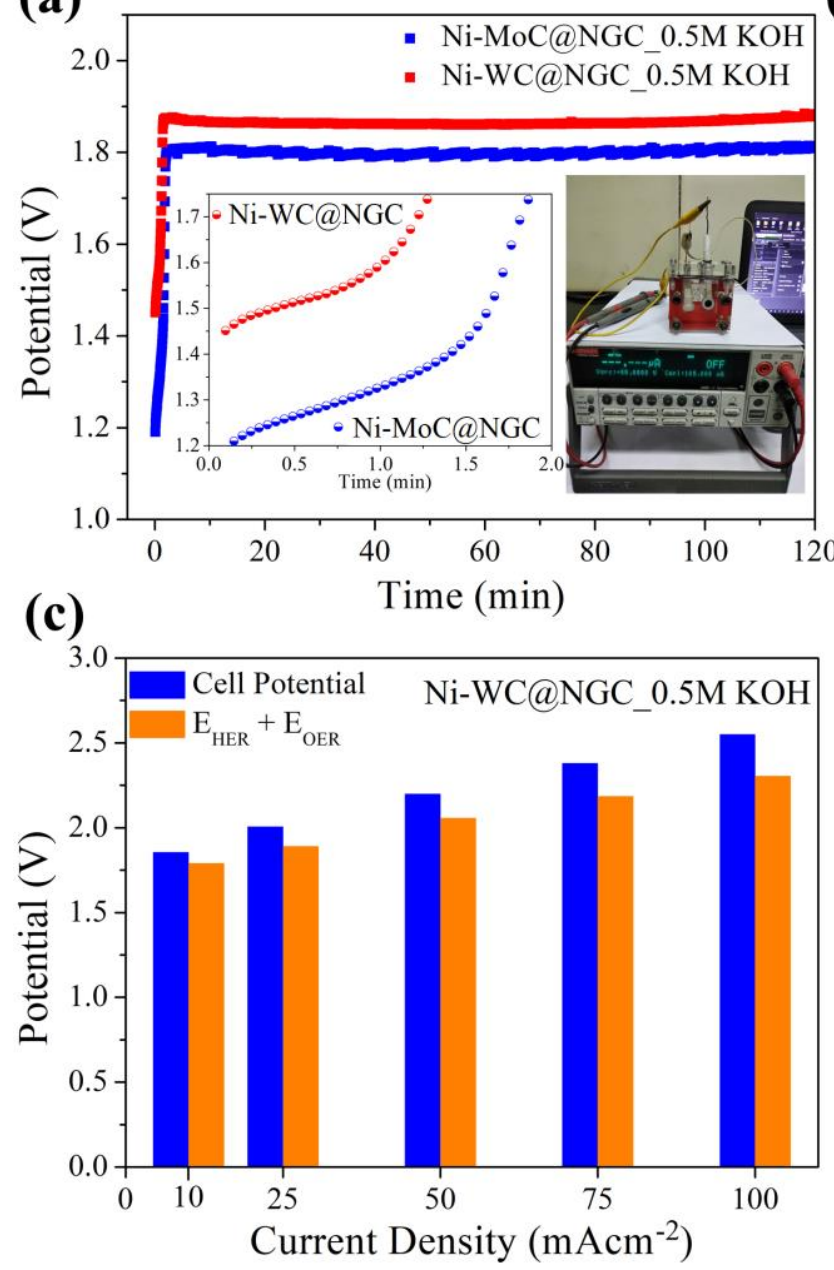

Ni-MoC: $60 \Omega$ ) measured at $1.6 \mathrm{~V}$ (vs. RHE, $\sim 20 \mathrm{~mA} / \mathrm{cm}^{2}$ ) decreases by $\sim$ one-third from $\mathrm{IrO}_{2}(188 \Omega)$ suggesting a faster electron transfer process for hydroxyl anion oxidation in (Figure 3f). Ni-MoC@NGC exhibited superior electrocatalytic OER activity as compared to many of the recently reported OER catalysts, operating under similar electrochemical conditions (Table S6). Both the compounds showed robust OER stability under dynamic potential cycling ( $1.3 \mathrm{~V}$ to $1.7 \mathrm{~V}$ vs RHE), with no degradation in activity after 1000 cycles of ADT as shown in Figure S13b, c. Ni is known to be very active during OER, ${ }^{77}$ and thus unlike in HER, is expected to participate as the active centre along with the W/Mo sites. The oxidized $\mathrm{Ni}$ atoms forms $\mathrm{Ni}$ (oxy)hydroxide species which is believed to be the actual catalytic centre for OER. ${ }^{17,77,78}$ This can be proved from the comparison of XANES spectra during and after electrochemistry (see the mechanism section), which shows a pronounced increase in white line intensity associated with the operando oxidation of Ni sites to the Ni-(oxy)hydroxide species. The identification of the $\mathrm{Ni}-\mathrm{OOH}$ phase from PXRD is tricky due to substantially low $\mathrm{Ni}$ content and its low crystallinity owing to fast in-situ formation. ${ }^{17}$

\subsection{Overall water splitting}

To investigate their potential as bifunctional OWS catalysts, commercial and customized GCE was used in a two electrode setup (Figure 4a, inset). The OWS studies were done in $0.5 \mathrm{M}$ $\mathrm{KOH}$ at current densities of 7, 10, 25, 50, 75, $100 \mathrm{~mA} / \mathrm{cm}^{2}$. The overall cell potentials $\left(\mathrm{E}_{\text {cell }}\right)$ obtained experimentally at various current densities under the two-electrode setup, are almost equal to the calculated $\mathrm{E}_{\text {cell }}$ as the sum of the cathodic and anodic potentials obtained from the HER/OER polarization curves (Figure 4b-c, Table S7). The $\mathrm{E}_{\text {cell }}$ at onset of water splitting, depicted as a hump in the upward I-V curve in Figure 4a, was found to be $1.34 \mathrm{~V}$ for Ni-MoC@NGC and 1.4 V for Ni-WC@NGC, which are very close to the thermodynamic potential $(1.23 \mathrm{~V})$ required for water splitting.

(b)

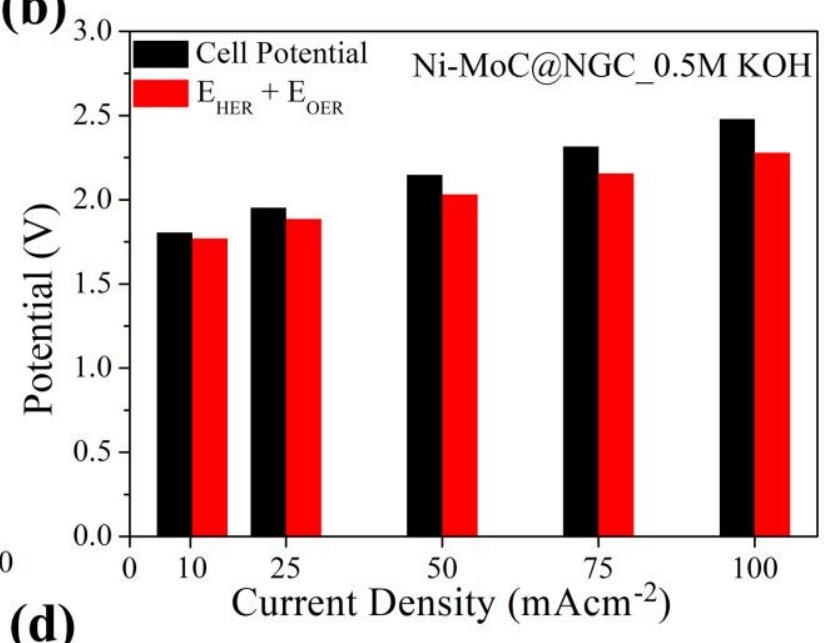

(d)

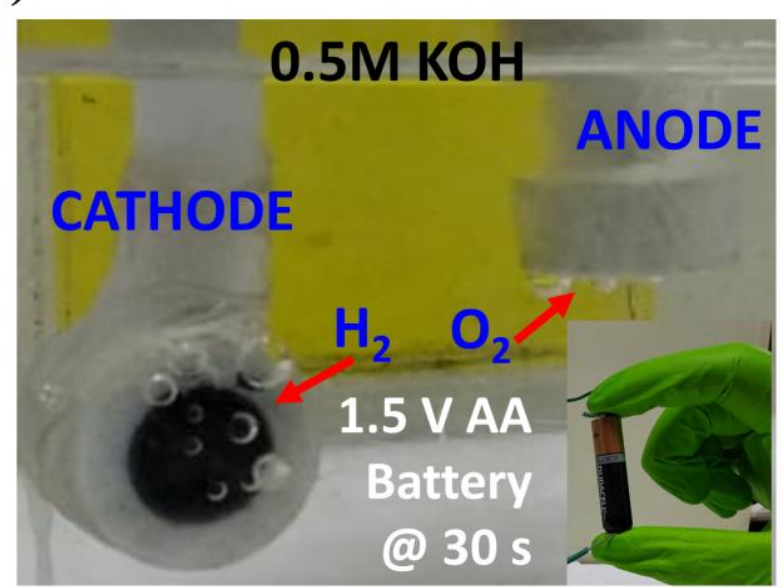

Figure 4. Overall water splitting activity of Ni-WC@NGC \& Ni-MoC@NGC in 0.5 M KOH: (a) Comparative overall water splitting activity and stability of Ni-WC\|Ni-WC \& Ni-MoC\|Ni-MoC electrolyzers @ $10 \mathrm{~mA} / \mathrm{cm}^{2}$ powered by a Keithly Source Meter. The comparison of overall cell potentials obtained experimentally at various current densities under the two electrode setup with the calculated $E_{\text {cell }}$ as the sum of the cathodic and anodic potentials at those current densities during individual HER/OER activity in $0.5 \mathrm{M} \mathrm{KOH}$ for (b) NiMoC@NGC and (c) Ni-WC@NGC. (d) The evolution of $\mathrm{H}_{2}$ and $\mathrm{O}_{2}$ gas bubbles at the cathode and anode (single chamber cell, two electrode setup) respectively aided by a $1.5 \mathrm{~V}$ AAA commercial battery, after $30 \mathrm{sec}$ of voltage application. 
The cell voltages@10 mA/ $\mathrm{cm}^{2}$ are obtained to be $1.75 \mathrm{~V} \&$ $1.8 \mathrm{~V}$ for the Ni-MoC@NGC and Ni-WC@NGC electrolyzers respectively, which are very close to the experimental $\mathrm{E}_{\text {cell }}$ value $(1.68 \mathrm{~V})$ observed for the combined state-of the-art catalysts $\left(\mathrm{Pt} / \mathrm{CllIO}_{2}\right)$ system, with $20 \% \mathrm{Pt} / \mathrm{C}$ as the cathode and $\mathrm{IrO}_{2}$ as the anode. Interestingly, the lowest cell potential of $1.60 \mathrm{~V}$ is exhibited by $20 \% \mathrm{Pt} / \mathrm{C}$ as the both cathodic and anodic catalyst. Furthermore, controlled experiments using different combinations of cathode and anode (Table S4) reveal that the HER in alkaline media is the limiting faradaic process for both the catalysts, as using $[\mathrm{Pt} / \mathrm{C} \| \mathrm{Ni}$ $\mathrm{MoC}$ or Ni-WC] we can easily achieve an Ecell of $1.6 \mathrm{~V}$ (Table S7). This highlights the excellent OER activity of the substituted catalysts under total water splitting conditions. Interestingly, for $\mathrm{Ni}-\mathrm{MoCl} \| \mathrm{Pt} / \mathrm{C}$ electrolyzer, the obtained $\mathrm{E}_{\text {cell }}$ is $2.06 \mathrm{~V}$ whereas, that for $\mathrm{Ni}-\mathrm{WC} \| \mathrm{Pt} / \mathrm{C}$ system is $2.15 \mathrm{~V}$ which indicate that both HER and OER are getting limited by Ni-MoC@NGC/NiWC@NGC at the cathode and Pt/C at the anode respectively. PtwirelPt-wire coupled electrolyzer requires a cell potential of $2.1 \mathrm{~V}$ to reach the same $\mathrm{H}_{2} \mathrm{O}$ splitting current density of $10 \mathrm{~mA} / \mathrm{cm}^{2}$. The durability of the Ni substituted WC@NGC and MoC@NGC catalysts were tested under the overall water splitting conditions for 2 hours, where both the catalysts exhibited a constant potential with negligible increase in $\mathrm{E}_{\text {cell. }}$ The Faradaic efficiencies (FE) for HER and OER of the Ni-MoC@NGC catalyst in the overall water splitting mode were obtained as $100 \%$ using a customized cell (Figure S14a and Video S1).

Finally to explore the practical applicability of the bifunctional Ni-MoC@NGC catalyst, a commercial 1.5 V AAA battery was used to split $\mathrm{H}_{2} \mathrm{O}$ in $0.5 \mathrm{M} \mathrm{KOH}$. Figure 4d, S14b and and Video S2 show the simultaneous and continuous hydrogen and oxygen evolution at the cathode and anode respectively in the $\mathrm{Ni}$ MoC@NGCINi-MoC@NGC electrolyzer powered by a $1.5 \mathrm{~V}$ commercial battery. Thus, the combined OWS studies on the Nisubstituted carbides demonstrate their tremendous potential for practical OWS applications. They can be further used as a case study for related non-noble metal systems depicting high electrocatalytic enhancement upon promotional secondary TM substitution.

\subsection{Mechanistic Analyses}

The electronic effect of $\mathrm{Ni}$ substitution and mechanistic understanding of activity enhancement is elucidated through density functional theory (DFT) analyses, which is corroborated by a series of systematic ex-situ (XPS, PXRD, discussed in earlier section) and in-situ XANES studies. HBE (hydrogen binding
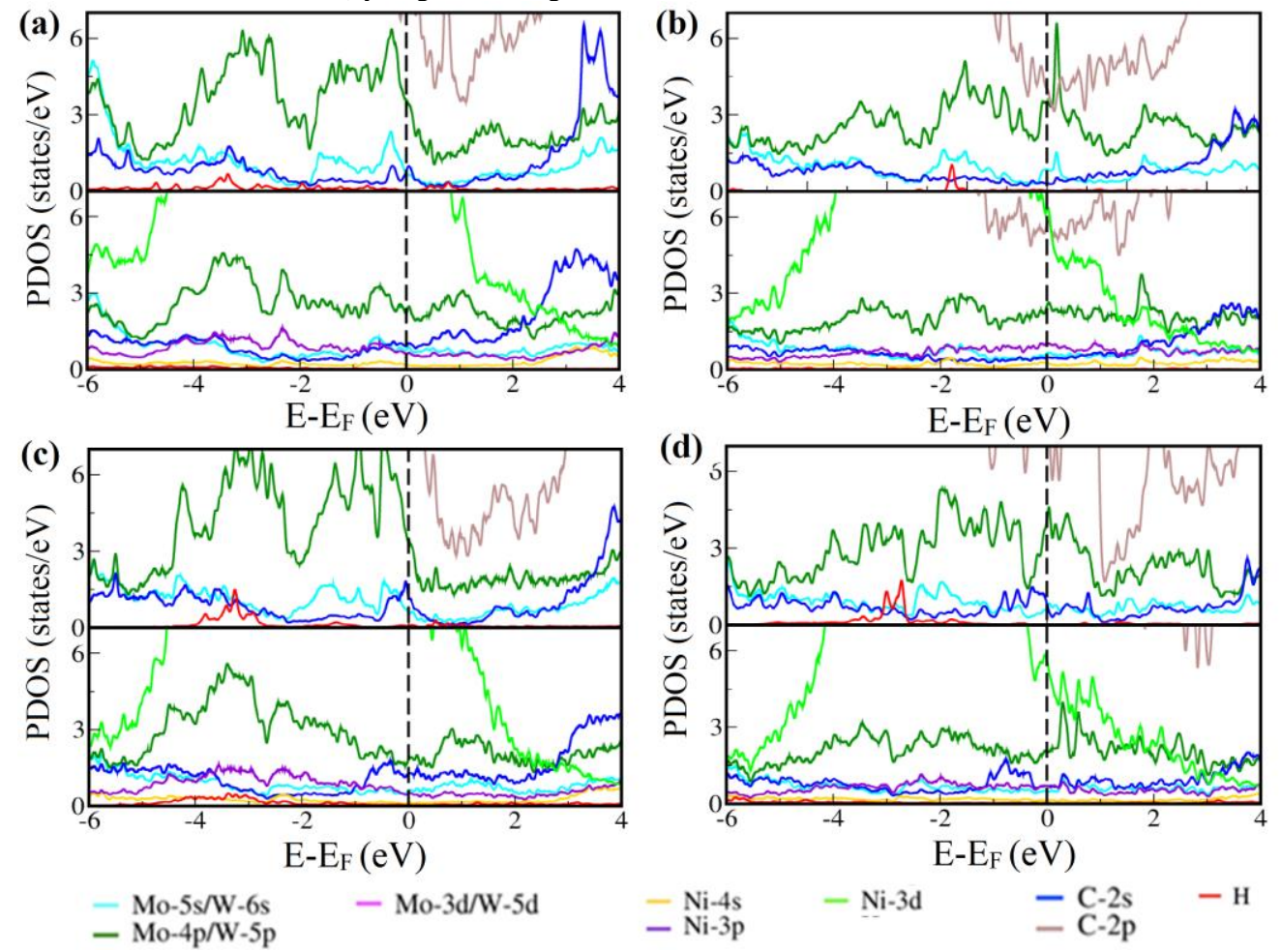

Figure 5. Projected density of states (PDOS) of structures with $\mathrm{H}$-adsorbed on pristine (top) and Ni-Mo/WC(1:5) substituted surfaces (bottom) of (a) MoC (100), (b) MoC (101), (c) WC (100) and (d) WC (101) surfaces. Dashed black lines represent the Fermi level ( $\left.E_{F}\right)$. PDOS (zoomed out) including all the orbitals is shown in Figure S18. 
For pristine MoC (100) surface, the adsorbate orbital is degenerate (Figure 5a), partially occupied and has energy just below the Fermi level $\left(\mathrm{E}_{\mathrm{F}}\right)$. The broad and split peak associated with HOAO shows a covalent interaction between $\mathrm{H}$ and the surface. PDOS of $\mathrm{H}$-atom adsorbed on pristine MoC (101) surface exhibits a sharp HOAO peak at energy below EF (Figure 5b) denoting more ionic nature of its interaction with the surface involving charge transfer. The $\mathrm{HOAO}$ of $\mathrm{H}$-atom adsorbed on $\mathrm{Ni}-$ $\operatorname{MoC}(1: 5)$ (100) and (101) surfaces is deeper in energy than that on pristine surfaces (Figure 5a, 5b) showing that $\mathrm{H}$-atom interacts more weakly with the Ni-substituted surfaces. HOAO of $\mathrm{H}$ adsorbed on pristine WC (100)/(101) surfaces occurs at energy just below $\mathrm{E}_{\mathrm{F}}$ and is partially occupied being degenerate with the W-6s and C-2s orbitals of the catalyst (Figure 5c, 5d). As a result, it resonates and facilitates charge transfer from the catalysts' surfaces. H-atom adsorbed on Ni-WC(1:5) (100) exhibits a much broader HOAO peak (Figure 5c), while the HOAO peak of $\mathrm{H}$ adsorbed on Ni-WC(1:5) (101) lies much lower in energy (Figure 5c, 5d), as expected. We estimated work functions $(\varphi)$, a descriptor relevant to catalytic activity of metals towards water reduction reaction, ${ }^{80}$ of the pristine, $\mathrm{Ni}-\mathrm{Mo} / \mathrm{WC}(1: 5)$ substituted (100) and (101) surfaces using the following relation:

$$
\varphi=V_{v a c}-E_{F}
$$

where, $V_{\text {vac }}$ is the potential energy in vacuum estimated from coarse-grained planar average of electrostatic potential. Water reduction potential is $-4.44 \mathrm{~V}$ with respect to vacuum. As evident in Table S10, the work functions of WC and MoC surfaces correspond to $\mathrm{E}_{\mathrm{F}}$ much below the hydrogen evolution potential. $\mathrm{Ni}$ substitution results in bringing shift in $\mathrm{EF}_{\mathrm{F}}$ closer to the HER potential, showing their viability as electrocatalysts. In summary, Ni-substitution in MCs permits engineering of Fermi surface/energy to weaken their interaction with $\mathrm{H}$.

When $\mathrm{Ni}$ in +2 oxidation state substitutes $\mathrm{W}$ or Mo (in +4 oxidation state) in the carbide lattice, ${ }^{81}$ an incumbent need to maintain the charge balance can trigger the Mo and $\mathrm{W}$ to attain a higher partial oxidation state, as observed from the XPS (Figure S4a, b, S4b) and XANES spectra (Figure S19a-b). XANES measurements at the Ni (K-edge, $8.33 \mathrm{keV}$ ), Mo (K-edge, 19.99 $\mathrm{keV}$ ) and $\mathrm{W}$ (LIII edge, $10.207 \mathrm{keV}$ ) edges (Figure S19a, S19b, and Figure 6) show a prominent increase in the white line intensity of the Mo/W centres upon Ni substitution (Figure S19a, S19b) which is indicative of the increase in unoccupied states of the d-orbitals., ${ }^{43,82}$ Interestingly, the Mo edge shows the appearance of an edge peak (Figure S19b), corresponding to an $1 \mathrm{~s} \rightarrow 4 p$ transition, presumably arising from a breaking in symmetry around the local coordination of Mo centres upon $\mathrm{Ni}$ substitution. The possibility of charge transfer between the (a)

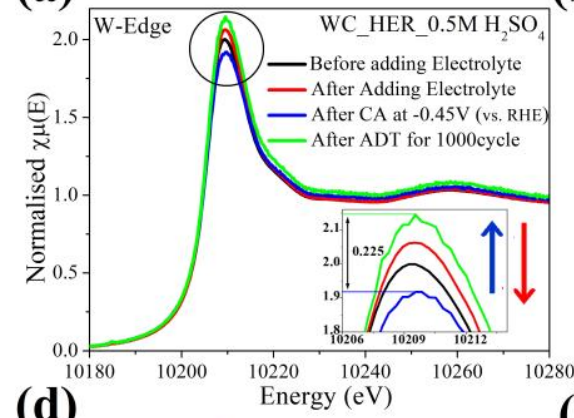

(b)
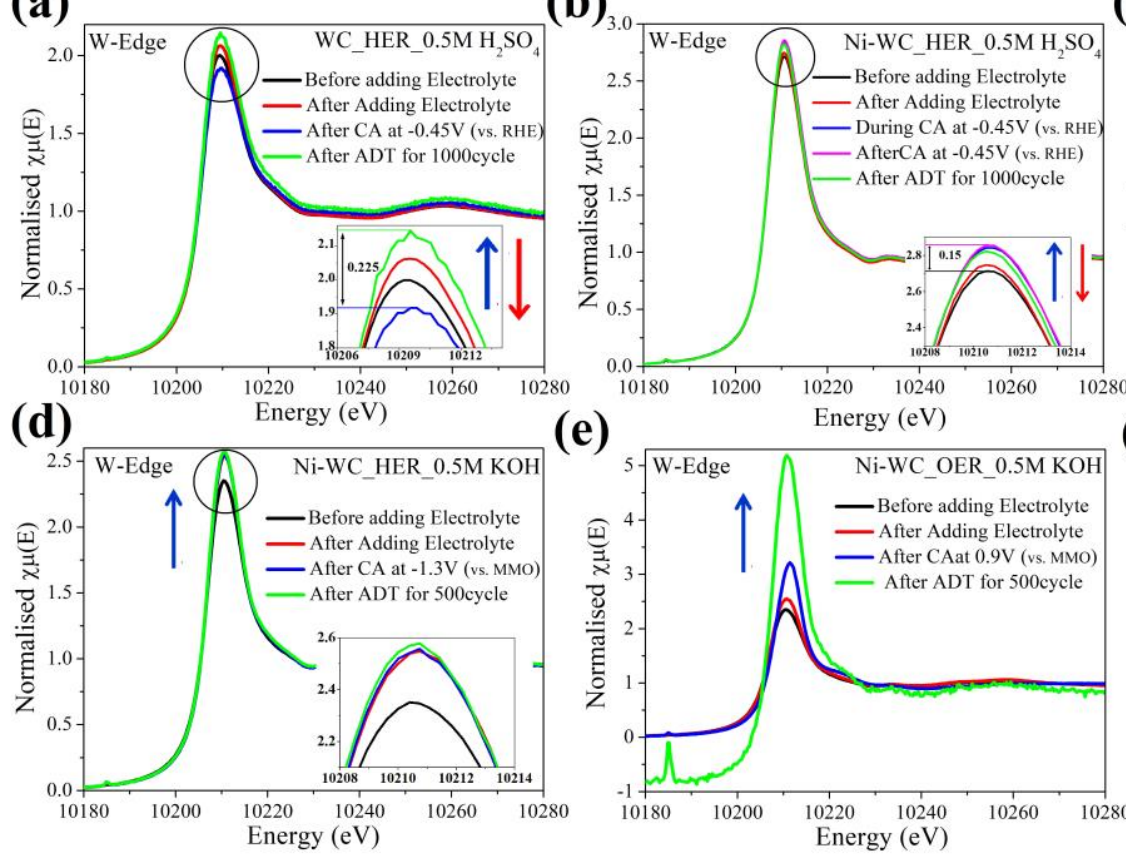

(e)

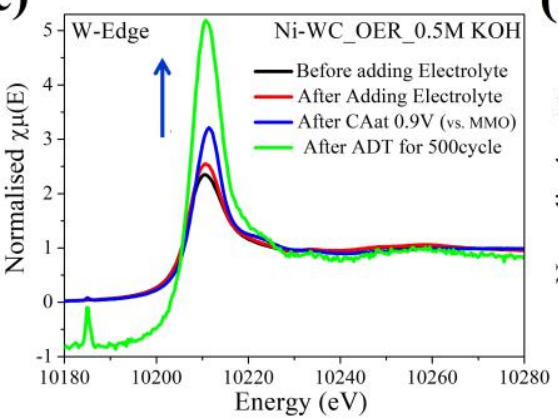

electronically linked Ni \& W/Mo ions is expected to optimize the electrochemical adsorption desorption processes resulting in enhanced electrocatalytic activity.

To understand the mechanistic effect of $\mathrm{Ni}$ induced electronic modulation of $\mathrm{W}$ and Mo centres on the real-time electrocatalytic activity, operando quick-EXAFS measurements are done in a customized in-situ setup as shown in Figure S20. In the unsubstituted WC, a gradual increase in white line intensity in the normalized W-edge XANES spectra is observed during electrochemistry and cycling (Figure 6a). This suggests that during HER, the active site, $\mathrm{W}$, has to attain a higher oxidation state for the electron transfer process of proton reduction which facilitates the electron transfer process from the electrode surface to the adsorbed $\mathrm{H}$ atom. Since in case of Ni-substituted WC, the W sites already exists at a partially higher oxidation state, the increase in white line intensity during electrochemistry is much lower $(0.15$ compared to 0.23 in WC@NGC) (Figure 6b), thereby allowing a much faster and enhanced electron transfer kinetics. Furthermore, during electrochemistry, the existence of a clear charge transfer process from $\mathrm{W}$ to $\mathrm{Ni}$ is evident from the corresponding decrease in white line intensity in the $\mathrm{Ni}$ edge (Figure 6c). This further facilitates the partial oxidation of $\mathrm{W}$ centre resulting in a more facile electron transfer process (Figure S21). As expected, W centres in WC@NGC and Ni-WC@NGC exhibit a contrasting variation in their chemical states after the $1 \mathrm{hr}-\mathrm{CA}$ at the negative potentials. Figure 6a and $6 \mathbf{b}$ shows that after $1 \mathrm{hr}-\mathrm{CA}, \mathrm{W}$ in case of the pristine WC suffers a substantial decrease in the white line intensity, while that in case of Ni-substituted system remains stabilised at the higher oxidation state. This proves, beyond doubt, that the in-situ charge transfer interaction between $\mathrm{Ni}$ and $\mathrm{W}$, helps the active $\mathrm{W}$ centre to achieve and maintain the desirable unfilled d-states, leading to a facile and stable electron transfer process during HER. The normalized in-situ XANES spectra of the NiWC@NGC catalyst during HER in basic media is shown in Figure 6d. The W-edge experiences an increase in the white line intensity on exposure to the $0.5 \mathrm{M} \mathrm{KOH}$ solution, which results from a partial surface oxidation of the WC by the action of hydroxyl anions. The increase in white line intensity during further HER process is less pronounced than that in acidic media which can be attributed to two reasons. Firstly, in basic media the extent of activity is somewhat hindered (at the same potential $\sim-0.45 \mathrm{~V}$ vs RHE) by the sluggish water activation kinetics leading to a lower current density. Secondly, the adsorbed species in alkaline media happens to be $\mathrm{H}-\mathrm{OH}^{*}$, in contrast to the $\mathrm{H}^{*}$ in acid, where $\delta(-\mathrm{ve}) \mathrm{O}$ atom is expected to bind to the metallic $\mathrm{W}$ site. The proton reduction after water activation thus, can be presumed to occur on the $\mathrm{C}$ site, or from a spilled over metallic site.

(c)
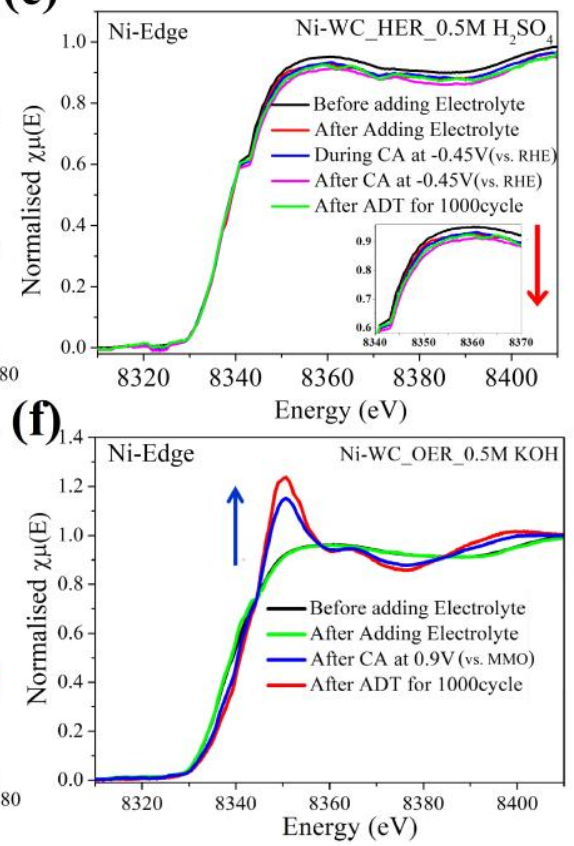

Figure 6. In-situ XANES studies at (a) W edges of WC@NGC during HER in 0.5 $\mathrm{M} \mathrm{H}_{2} \mathrm{SO}_{4}$, (b) W edges of Ni-WC@ NGC during HER in in $0.5 \mathrm{M} \mathrm{H}_{2} \mathrm{SO}_{4}$, (c) Ni edges of Ni-WC@ NGC during HER in $0.5 \mathrm{M} \mathrm{H}_{2} \mathrm{SO}_{4}$, (d) W edges of Ni-WC@ NGC during HER in 0.5M KOH, (e) W edges of Ni-WC@NGC during OER in 0.5M KOH and (f) Ni edges of Ni-WC@NGC during OER in 0.5M KOH. 
This suffices a moderate increase in $\mathrm{W}$ oxidation state during alkaline HER. The slight increase in $\mathrm{W}$ oxidation state is facilitated by a charge transfer process from $\mathrm{W}$ to $\mathrm{Ni}$, like in the previous case, which was reflected in the decrease in white line intensity of Ni edge (Figure S19c, d).

Oxygen evolution in alkaline media occurs at a much higher potential, where the oxidation of the $\mathrm{W}$ and $\mathrm{Ni}$ centres becomes inevitable under constant operations. Thus the normalized XANES spectra for both $\mathrm{W}$ and Ni centres after ADT and CA exhibits a high increase in the white line intensities, as shown in Figure 6e, f. This can also be explained from the Pourbaix diagram of the W and $\mathrm{Ni}$ species which predicts the existence of higher oxidation state of the elements at the corresponding $\mathrm{pH}$ (13-14) and potential ranges ( 0.6 to $1 \mathrm{~V}$ vs $\mathrm{RHE}) .{ }^{83} \mathrm{Ni}$ is speculated to play a role, alongside $\mathrm{W}$, as an active centre in OER through the formation of the well-known $\mathrm{Ni}-\mathrm{OOH}$ species in alkaline media as evident from the change in post edge XAS spectra of the Ni-edge during OER (Figure S21).

\section{Concluding Remarks}

In summary, we have designed and presented two nanocomposites of hexagonal Ni-W/MoC carbide nanoparticles grafted on NGC support, synthesized through a facile one-step in-situ carburization technique. Both the nano-hybrids performed excellently as cheap, highly efficient and stable electrocatalysts for hydrogen \& oxygen evolution and overall water splitting. The superior activity of Ni-MoC@NGC for HER, OER and overall water splitting surpasses most of the reported carbide or non-noble metal systems, which is further showcased in the $1.5 \mathrm{~V}$ battery aided OWS activity. The $\mathrm{Ni}$ induced modulation of carbide electronic structure was proved through various techniques like XPS, XAS and DFT calculations. The colossal enhancement in activity is shown to stem from the multifunctional role of the $\mathrm{Ni}$ substituent, which on one hand enhances the kinetic factors like ECSA, and on the other, controls the thermodynamic aspects like intermediate adsorption energies, oxidation state modulation of the active centres and electronic DOS. Real time mechanistic analyses through operando XAS studies reveals that the synergistic electronic interactions through in-situ charge transfer between $\mathrm{Ni}$ and active centres promotes the HER kinetics. During OER Ni was found to play an active role in catalytic enhancement through the formation of Ni-oxyhydroxide species. The new mechanistic insights elucidated in this work will help in re-understanding the concepts of substituent induced synergistic enhancement of water splitting activity in a wide variety of electrocatalysts.

\section{Acknowledgements}

Financial support from Department of Science and Technology (DST) (HFC-2018 Grant No.DST/TMD/HFC/2K18/128(G)), Technical Research Centre (TRC) (Grant Number: JNC/AO/DSTTRC/C.14.10/16-2750), Jawaharlal Nehru Centre for Advanced Scientific Research (JNCASR) is gratefully acknowledged. SR and DB thank Council of Scientific and Industrial Research for their fellowship. LD, SCS and VR thank JNCASR for their research fellowship. SCP thanks DST for SwarnaJayanti Fellowship (Grant Number: DST/SJF/CSA-02/2017-18).

References

1. Roy, S., Cherevotan, A. \& Peter, S. C. Thermochemical $\mathrm{CO}_{2}$ Hydrogenation to Single Carbon Products: Scientific and Technological Challenges. ACS Energy Lett. 3, 1938-1966 (2018).

2. Roy, S. \& Peter, S. C. in Adv. Chem. Phys. Mater. 399-428.

3. Wu, H. B., Xia, B. Y., Yu, L., Yu, X. Y. \& Lou, X. W. Porous molybdenum carbide nano-octahedrons synthesized via confined carburization in metal-organic frameworks for efficient hydrogen production. Nat. Commun. 6, 6512-6519 (2015).

4. $\mathrm{Xu}, \mathrm{Y}$. T. et al. Cage-Confinement Pyrolysis Route to Ultrasmall Tungsten Carbide Nanoparticles for Efficient Electrocatalytic Hydrogen Evolution. J. Am. Chem. Soc. 139, 5285-5288 (2017).

5. Yuan-Yuan Ma, Z.-L. L., Li-Kai Yan, Yong-Hui Wang, HuaQiao Tan, Kun Feng, Yu-Jian Xia, Jun Zhong, Yang Liu, Zhen-Hui Kang and Yang-Guang Li. Highly efficient hydrogen evolution triggered by a multi-interfacial Ni/WC hybrid electrocatalyst. Energy Environ. Sci. 11, 2114-2123 (2018).

6. Li, J. S. et al. Coupled molybdenum carbide and reduced graphene oxide electrocatalysts for efficient hydrogen evolution. Nat. Commun. 7, 11204-11212 (2016).
7. Gao, X. H. et al. Hierarchical $\mathrm{NiCo}_{2} \mathrm{O}_{4}$ Hollow Microcuboids as Bifunctional Electrocatalysts for Overall Water-Splitting. Angew. Chem. Inter. Ed. 55, 6290-6294 (2016).

8. Guo, Y. N. et al. Elaborately assembled core-shell structured metal sulfides as a bifunctional catalyst for highly efficient electrochemical overall water splitting. Nano Energy 47, 494502 (2018).

9. Zou, X. X. \& Zhang, Y. Noble metal-free hydrogen evolution catalysts for water splitting. Chem. Soc. Rev. 44, 5148-5180 (2015).

10. Chen, W. F. et al. Highly active and durable nanostructured molybdenum carbide electrocatalysts for hydrogen production. Energy Environ. Sci. 6, 943-951 (2013).

11. Yan, Y., Xia, B. Y., Zhao, B. \& Wang, X. A review on noblemetal-free bifunctional heterogeneous catalysts for overall electrochemical water splitting. J Mater. Chem. A 4, 1758717603 (2016).

12. Xiong, B. Y., Chen, L. S. \& Shi, J. L. Anion-Containing Noble-Metal-Free Bifunctional Electrocatalysts for Overall Water Splitting. ACS Catal. 8, 3688-3707 (2018).

13. Wang, J., Xu, F., Jin, H. Y., Chen, Y. Q. \& Wang, Y. NonNoble Metal-based Carbon Composites in Hydrogen Evolution Reaction: Fundamentals to Applications. Adv. Mater. 29, 1605838-1605873 (2017).

14. Du, P. W. \& Eisenberg, R. Catalysts made of earth-abundant elements $(\mathrm{Co}, \mathrm{Ni}, \mathrm{Fe})$ for water splitting: Recent progress and future challenges. Energy Environ. Sci. 5, 6012-6021 (2012).

15. Mukherjee, D., Austeria, P. M. \& Sampath, S. TwoDimensional, Few-Layer Phosphochalcogenide, FePS 3 : A New Catalyst for Electrochemical Hydrogen Evolution over Wide pH Range. ACS Energy Lett. 1, 367-372 (2016).

16. Hunt, S. T., Milina, M., Wang, Z. S. \& Roman-Leshkov, Y. Activating earth-abundant electrocatalysts for efficient, lowcost hydrogen evolution/oxidation: sub-monolayer platinum coatings on titanium tungsten carbide nanoparticles. Energy Environ. Sci. 9, 3290-3301 (2016).

17. Kong, D. et al. 3D hierarchical defect-rich $\mathrm{NiMo}_{3} \mathrm{~S}_{4}$ nanosheet arrays grown on carbon textiles for high-performance sodiumion batteries and hydrogen evolution reaction. Nano Energy 49, 460-470 (2018).

18. Shi, Y. M. \& Zhang, B. Recent advances in transition metal phosphide nanomaterials: synthesis and applications in hydrogen evolution reaction (vol 45, pg 1781, 2016). Chem. Soc. Rev. 45, 1781-1781 (2016).

19. Dutta, A. \& Pradhan, N. Developments of Metal Phosphides as Efficient OER Precatalysts. J Phys. Chem. Lett. 8, 144-152 (2017).

20. Tan, Y. et al. Versatile nanoporous bimetallic phosphides towards electrochemical water splitting. Energy Environ. Sci. 9, 2257-2261 (2016).

21. Stern, L.-A., Feng, L., Song, F. \& Hu, X. $\mathrm{Ni}_{2} \mathrm{P}$ as a Janus catalyst for water splitting: the oxygen evolution activity of $\mathrm{Ni}_{2} \mathrm{P}$ nanoparticles. Energy Environ. Sci. 8, 2347-2351 (2015).

22. Michalsky, R., Zhang, Y.-J. \& Peterson, A. A. Trends in the Hydrogen Evolution Activity of Metal Carbide Catalysts. ACS Catal. 4, 1274-1278 (2014).

23. Ma, Y. F., Guan, G. Q., Hao, X. G., Cao, J. \& Abudula, A. Molybdenum carbide as alternative catalyst for hydrogen production - A review. Renew. Sust. Energ. Rev. 75, 11011129 (2017).

24. Liao, L. et al. A nanoporous molybdenum carbide nanowire as an electrocatalyst for hydrogen evolution reaction. Energy Environ. Sci. 7, 387-392 (2014).

25. Balogun, M. S. et al. Updates on the development of nanostructured transition metal nitrides for electrochemical energy storage and water splitting. Mater. Today 20, 425-451 (2017).

26. Abghoui, Y. \& Skulason, E. Hydrogen Evolution Reaction Catalyzed by Transition-Metal Nitrides. J Phys. Chem. C 121, 24036-24045 (2017).

27. Xie, J. F. \& Xie, Y. Transition Metal Nitrides for Electrocatalytic Energy Conversion: Opportunities and Challenges. Chem. Eur. J. 22, 3588-3598 (2016).

28. Chen, L. et al. Metal boride better than Pt: $\mathrm{HCP} \mathrm{Pd}_{2} \mathrm{~B}$ as a superactive hydrogen evolution reaction catalyst. Energy Environ. Sci. 12, 3099-3105 (2019). 
29. Wang, F. M. et al. Recent advances in transition-metal dichalcogenide based nanomaterials for water splitting. Nanoscale 7, 19764-19788 (2015).

30. Long, X., Lin, H., Zhou, D., An, Y. \& Yang, S. Enhancing Full Water-Splitting Performance of Transition Metal Bifunctional Electrocatalysts in Alkaline Solutions by Tailoring $\mathrm{CeO}_{2}$-Transition Metal Oxides-Ni Nanointerfaces. ACS Energy Lett. 3, 290-296 (2018).

31. Anantharaj, S. et al. Recent Trends and Perspectives in Electrochemical Water Splitting with an Emphasis on Sulfide, Selenide, and Phosphide Catalysts of $\mathrm{Fe}, \mathrm{Co}$, and $\mathrm{Ni}$ : $\mathrm{A}$ Review. ACS Catal. 6, 8069-8097 (2016).

32. Zhou, Y. et al. Revealing the Contribution of Individual Factors to Hydrogen Evolution Reaction Catalytic Activity. Adv. Mater. 30, 1706076-1706085 (2018).

33. Sarma, S. C., Mishra, V., Ann Mary, K. A., Roy, S. \& Peter, S. C. "Inverse Strain Effect in Atomic Scale"-Enhanced Hydrogen Evolution Activity and Durability in $\mathrm{Cu}-$ Substituted Palladseite. ACS Energy Lett. 3, 3008-3014 (2018).

34. Joyner, J. et al. Graphene Supported $\mathrm{MoS}_{2}$ Structures with High Defect Density for an Efficient HER Electrocatalysts. ACS Appl. Mater. Inter. 12, 12629-12638 (2020).

35. Lukowski, M. A. et al. Highly active hydrogen evolution catalysis from metallic WS 2 nanosheets. Energy Environ. Sci. 7, 2608-2613 (2014).

36. Roger, I., Shipman, M. A. \& Symes, M. D. Earth-abundant catalysts for electrochemical and photoelectrochemical water splitting. Nat. Rev. Chem. 1, 0003 (2017).

37. $\mathrm{Yu}, \mathrm{L}$. et al. $\mathrm{Cu}$ nanowires shelled with $\mathrm{NiFe}$ layered double hydroxide nanosheets as bifunctional electrocatalysts for overall water splitting. Energy Environ. Sci. 10, 1820-1827 (2017).

38. Shen, Y., Li, L., Xi, J. Y. \& Qiu, X. P. A facile approach to fabricate free-standing hydrogen evolution electrodes: riveting tungsten carbide nanocrystals to graphite felt fabrics by carbon nanosheets. J Mater. Chem. A 4, 5817-5822 (2016).

39. Regmi, Y. N. et al. Carbides of group IVA, VA and VIA transition metals as alternative HER and ORR catalysts and support materials. J Mater. Chem. A 3, 10085-10091 (2015).

40. Wang, Q. et al. Design of active nickel single-atom decorated $\mathrm{MoS}_{2}$ as a pH-universal catalyst for hydrogen evolution reaction. Nano Energy 53, 458-467 (2018).

41. Regmi, Y. N. \& Leonard, B. M. General synthesis method for bimetallic carbides of group VIIIA first row transition metals with molybdenum and tungsten for fuel cell catalysis. Abstr. Pap. Am. Chem. S. 248 (2014).

42. Regmi, Y. N. \& Leonard, B. M. General Synthesis Method for Bimetallic Carbides of Group VIIIA First Row Transition Metals with Molybdenum and Tungsten. Chem. Mater. 26, 2609-2616 (2014).

43. Hunt, S. T., Kokumai, T. M., Zanchet, D. \& Román-Leshkov, Y. Alloying Tungsten Carbide Nanoparticles with Tantalum: Impact on Electrochemical Oxidation Resistance and Hydrogen Evolution Activity. J Phys. Chem. C 119, 1369113699 (2015).

44. Wan, C., Regmi, Y. N. \& Leonard, B. M. Multiple Phases of Molybdenum Carbide as Electrocatalysts for the Hydrogen Evolution Reaction. Angew. Chem. Inter. Ed. 53, 6407-6410 (2014).

45. Mu, Y. P. et al. Controllable synthesis of molybdenum carbide nanoparticles embedded in porous graphitized carbon matrixes as efficient electrocatalyst for hydrogen evolution reaction. Electrochim. Acta 215, 357-365 (2016).

46. Cheng, Z. H. et al. Interconnected Molybdenum CarbideBased Nanoribbons for Highly Efficient and Ultrastable Hydrogen Evolution. ACS Appl. Mater. Inter. 9, 24608-24615 (2017).

47. Gong, Q. F. et al. Ultrasmall and phase-pure $\mathrm{W}_{2} \mathrm{C}$ nanoparticles for efficient electrocatalytic and photoelectrochemical hydrogen evolution. Nat. Commun. 7, 13216-13224 (2016).

48. Dubouis, N. et al. Interfacial Interactions as an Electrochemical Tool To Understand Mo-Based Catalysts for the Hydrogen Evolution Reaction. ACS Catal. 8, 828-836 (2018).

49. Zhang, J. et al. Interface Engineering of $\mathrm{MoS}_{2} / \mathrm{Ni}_{3} \mathrm{~S}_{2}$ Heterostructures for Highly Enhanced Electrochemical
Overall-Water-Splitting Activity. Angew. Chem. Inter. Ed. 55, 6702-6707 (2016).

50. Esposito, D. V. \& Chen, J. G. Monolayer platinum supported on tungsten carbides as low-cost electrocatalysts: opportunities and limitations. Energy Environ. Sci. 4, 3900-3912 (2011).

51. Zhao, Y., Kamiya, K., Hashimoto, K. \& Nakanishi, S. Hydrogen Evolution by Tungsten Carbonitride Nanoelectrocatalysts Synthesized by the Formation of a Tungsten Acid/Polymer Hybrid In Situ. Angew. Chem. Inter. Ed. 52, 13638-13641 (2013).

52. Li, Z. S. et al. Hollow hemisphere-shaped macroporous graphene/tungsten carbide/platinum nanocomposite as an efficient electrocatalyst for the oxygen reduction reaction. Electrochim. Acta 221, 31-40 (2016).

53. Liu, Y. P. et al. Coupling $\mathrm{Mo}_{2} \mathrm{C}$ with Nitrogen-Rich Nanocarbon Leads to Efficient Hydrogen-Evolution Electrocatalytic Sites. Angew. Chem. Inter. Ed. 54, 1075210757 (2015).

54. Gomez-Marin, A. M. \& Ticianelli, E. A. Effect of transition metals in the hydrogen evolution electrocatalytic activity of molybdenum carbide. Appl. Catal. B-Environ. 209, 600-610 (2017).

55. Zhang, Y. et al. A Mn-doped $\mathrm{Ni}_{2} \mathrm{P}$ nanosheet array: an efficient and durable hydrogen evolution reaction electrocatalyst in alkaline media. Chem. Commun. 53, 1104811051 (2017).

56. Liu, T. T. et al. Mn Doping of CoP Nanosheets Array: An Efficient Electrocatalyst for Hydrogen Evolution Reaction with Enhanced Activity at All pH Values. ACS Catal. 7, 98102 (2017).

57. Liu, T. T. et al. Enhanced Electrocatalysis for Energy-Efficient Hydrogen Production over CoP Catalyst with Nonelectroactive $\mathrm{Zn}$ as a Promoter. Adv. Energy Mater. 7, 1700020-1700028 (2017).

58. Zhang, R. et al. Al-Doped CoP nanoarray: a durable watersplitting electrocatalyst with superhigh activity. Nanoscale $\mathbf{9}$, 4793-4800 (2017).

59. Norskov, J. K. et al. Trends in the exchange current for hydrogen evolution. J. Electrochem. Soc. 152, J23-J26 (2005).

60. Piontek, S. et al. Influence of the Fe:Ni Ratio and Reaction Temperature on the Efficiency of $\left(\mathrm{Fe}_{\mathrm{x}} \mathrm{Ni}_{1-\mathrm{x}}\right) 9 \mathrm{~S} 8$ Electrocatalysts Applied in the Hydrogen Evolution Reaction. ACS Catal. 8, 987-996 (2018).

61. Hammer, B., Norskov, J. K. Theoretical Surface Science and Catalysis-Calculations and Concepts. Adv. Catal. 45, 71-129 (2000).

62. Hammer, B., Scheffler, M., Jacobsen, K. W. \& Norskov, J. K. Multidimensional potential energy surface for $\mathrm{H}_{2}$ dissociation over Cu(111). Phys. Rev. Lett. 73, 1400-1403 (1994).

63. Wang, X. et al. Regulation of Coordination Number over Single Co Sites: Triggering the Efficient Electroreduction of $\mathrm{CO}_{2}$. Angew. Chem. Int. Ed. 57, 1944-1948 (2018).

64. Luo, J. et al. Water photolysis at $12.3 \%$ efficiency via perovskite photovoltaics and Earth-abundant catalysts. Science 345, 1593-1596 (2014).

65. $\mathrm{Yu}, \mathrm{L}$. et al. Amorphous NiFe layered double hydroxide nanosheets decorated on 3D nickel phosphide nanoarrays: a hierarchical core-shell electrocatalyst for efficient oxygen evolution. J Mater. Chem. A 6, 13619-13623 (2018).

66. Gong, M. \& Dai, H. J. A mini review of NiFe-based materials as highly active oxygen evolution reaction electrocatalysts. Nano Res. 8, 23-39 (2015).

67. Chen, M. H., Liu, J. L., Zhou, W. J., Lin, J. Y. \& Shen, Z. X. Nitrogen-doped Graphene-Supported Transition-metals Carbide Electrocatalysts for Oxygen Reduction Reaction. Sci. Rep. 5, 10389-10399 (2015).

68. Hou, Y. et al. Strongly Coupled 3D Hybrids of N-doped Porous Carbon Nanosheet/CoNi Alloy-Encapsulated Carbon Nanotubes for Enhanced Electrocatalysis. Small 11, 59405948 (2015).

69. Ma, Y. Y. et al. Highly efficient hydrogen evolution from seawater by a low-cost and stable CoMoP@C electrocatalyst superior to Pt/C. Energy Environ. Sci. 10, 788-798 (2017).

70. Tang, Y. J. et al. Molybdenum Disulfide/Nitrogen-Doped Reduced Graphene Oxide Nanocomposite with Enlarged Interlayer Spacing for Electrocatalytic Hydrogen Evolution. Adv. Energy Mater. 6, 1600116-1600123 (2016). 
71. Susi, T., Pichler, T. \& Ayala, P. X-ray photoelectron spectroscopy of graphitic carbon nanomaterials doped with heteroatoms. Beilstein J. Nanotechnol. 6, 177-192 (2015).

72. Kondo, T. et al. Observation of Landau levels on nitrogendoped flat graphite surfaces without external magnetic fields. Sci. Rep. 5, 16412-16420 (2015).

73. Choi, S. et al. Vacancy-engineered catalysts for water electrolysis. CrystEngComm 22, 1500-1513 (2020).

74. Laursen, A. B. et al. Electrochemical Hydrogen Evolution: Sabatier's Principle and the Volcano Plot. J. Chem. Educ. 89, 1595-1599 (2012).

75. Danilovic, N. et al. Enhancing the Alkaline Hydrogen Evolution Reaction Activity through the Bifunctionality of $\mathrm{Ni}(\mathrm{OH})_{2} /$ Metal Catalysts. Angew. Chem. Inter. Ed. 51, 1249512498 (2012).

76. Zhang, J. et al. Efficient hydrogen production on $\mathrm{MoNi}_{4}$ electrocatalysts with fast water dissociation kinetics. Nat. Commun. 8, 15437-15445 (2017).

77. Jamesh, M. I. Recent progress on earth abundant hydrogen evolution reaction and oxygen evolution reaction bifunctional electrocatalyst for overall water splitting in alkaline media. $J$. Power Sources 333, 213-236 (2016).

78. Jin, S. Are Metal Chalcogenides, Nitrides, and Phosphides Oxygen Evolution Catalysts or Bifunctional Catalysts? ACS Energy Lett. 2, 1937-1938 (2017).

79. Hinnemann, B. et al. Biomimetic Hydrogen Evolution: $\mathrm{MoS}_{2}$ Nanoparticles as Catalyst for Hydrogen Evolution. J. Am. Chem. Soc. 127, 5308-5309 (2005).

80. Pankajakshan, P. et al. Machine Learning and Statistical Analysis for Materials Science: Stability and Transferability of Fingerprint Descriptors and Chemical Insights. Chem. Mater. 29, 4190-4201 (2017).

81. Oshikawa, K., Nagai, M. \& Omi, S. Characterization of molybdenum carbides for methane reforming by TPR, XRD, and XPS. J. Phys. Chem. B 105, 9124-9131 (2001).

82. Feng, K. et al. $\mathrm{Cu}_{\mathrm{x}} \mathrm{Co}_{1-\mathrm{x}} \mathrm{O}$ Nanoparticles on Graphene Oxide as A Synergistic Catalyst for High-Efficiency Hydrolysis of Ammonia-Borane. Angew. Chem. Inter. Ed. 55, 11950-11954 (2016).

83. Takeno, N. Atlas of Eh-pH diagrams. Geological survey of Japan open file report 419, 102 (2005).

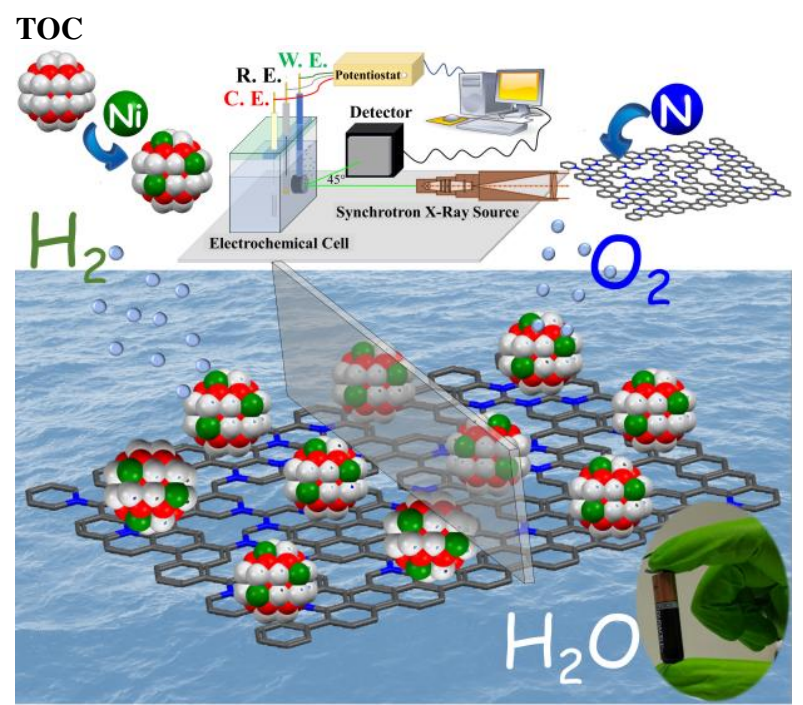




\section{Figures}
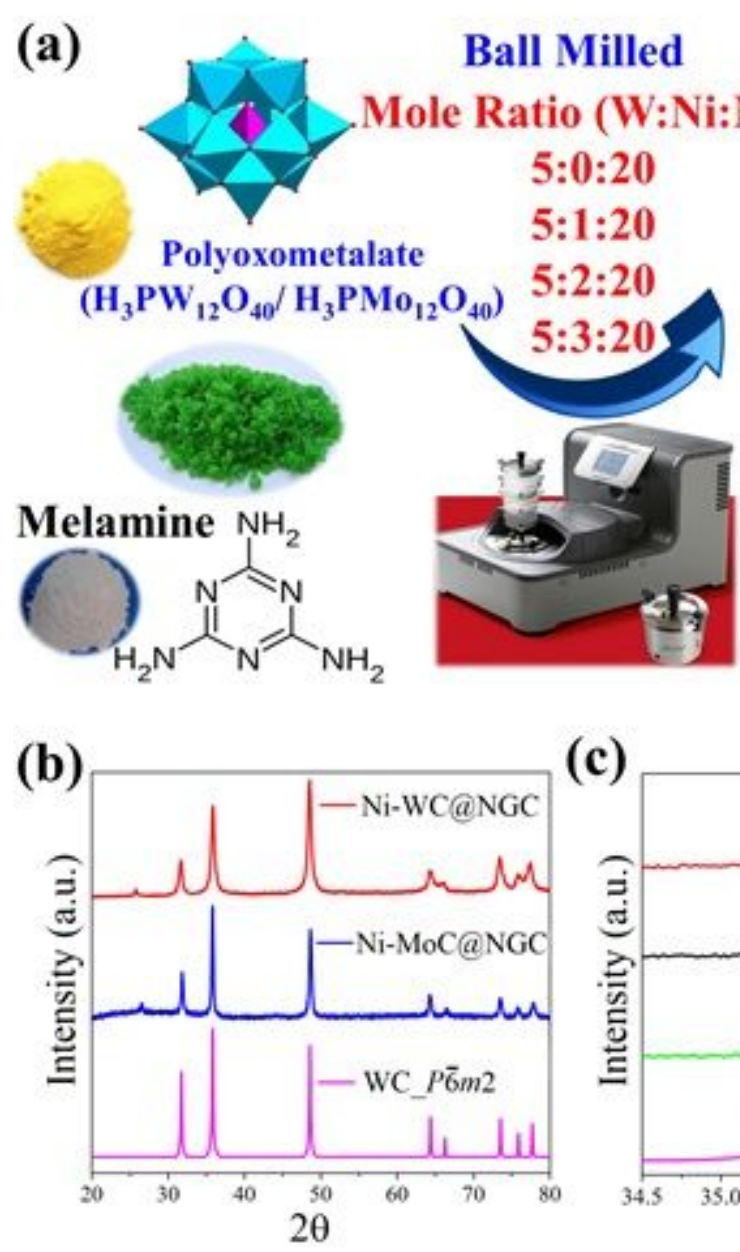

(c)

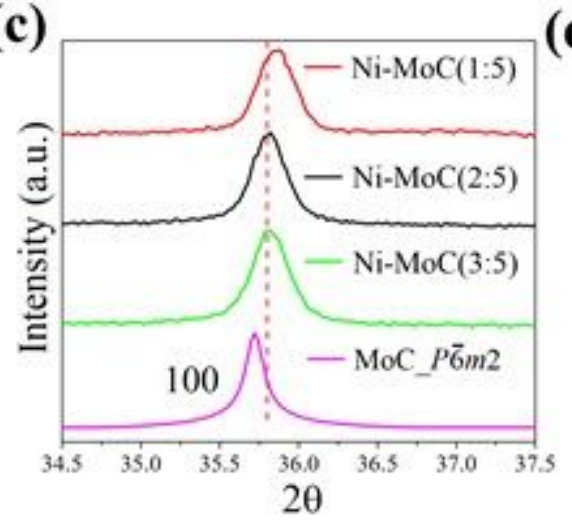

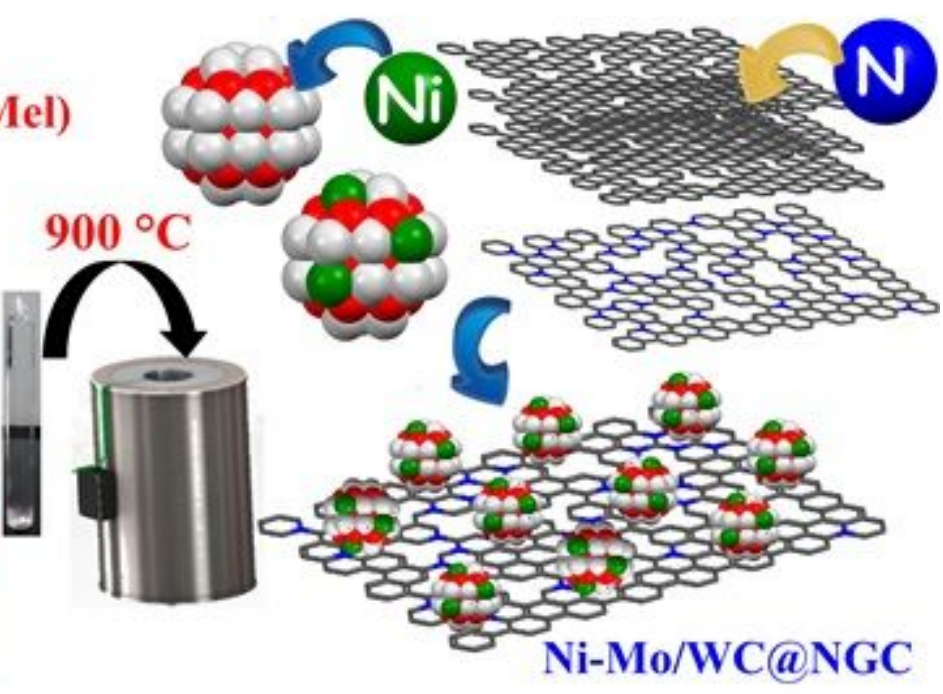

(d)

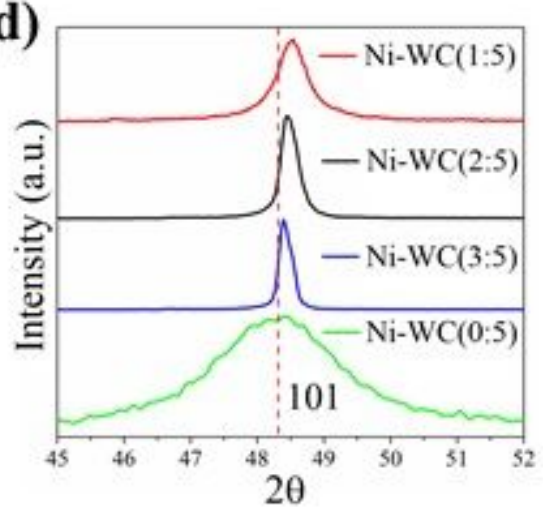

Figure 1

Synthesis and characterization of Ni-WC@NGC \& Ni-MoC@NGC: (a) Synthetic scheme for NiW/MoC@NGC showing different steps of precursor mixing, carbonization and Ni substituion into W/MoC lattice and N doping in carbon matrix, (b) Comparative PXRD patterns of Ni-WC@NGC \& Ni-MoC@NGC, (c) PXRD shift in 100 plane of Ni-MoC having different precursor mole ratios and (d) PXRD shift in 101 plane of Ni-MWC having different precursor mole ratios. 

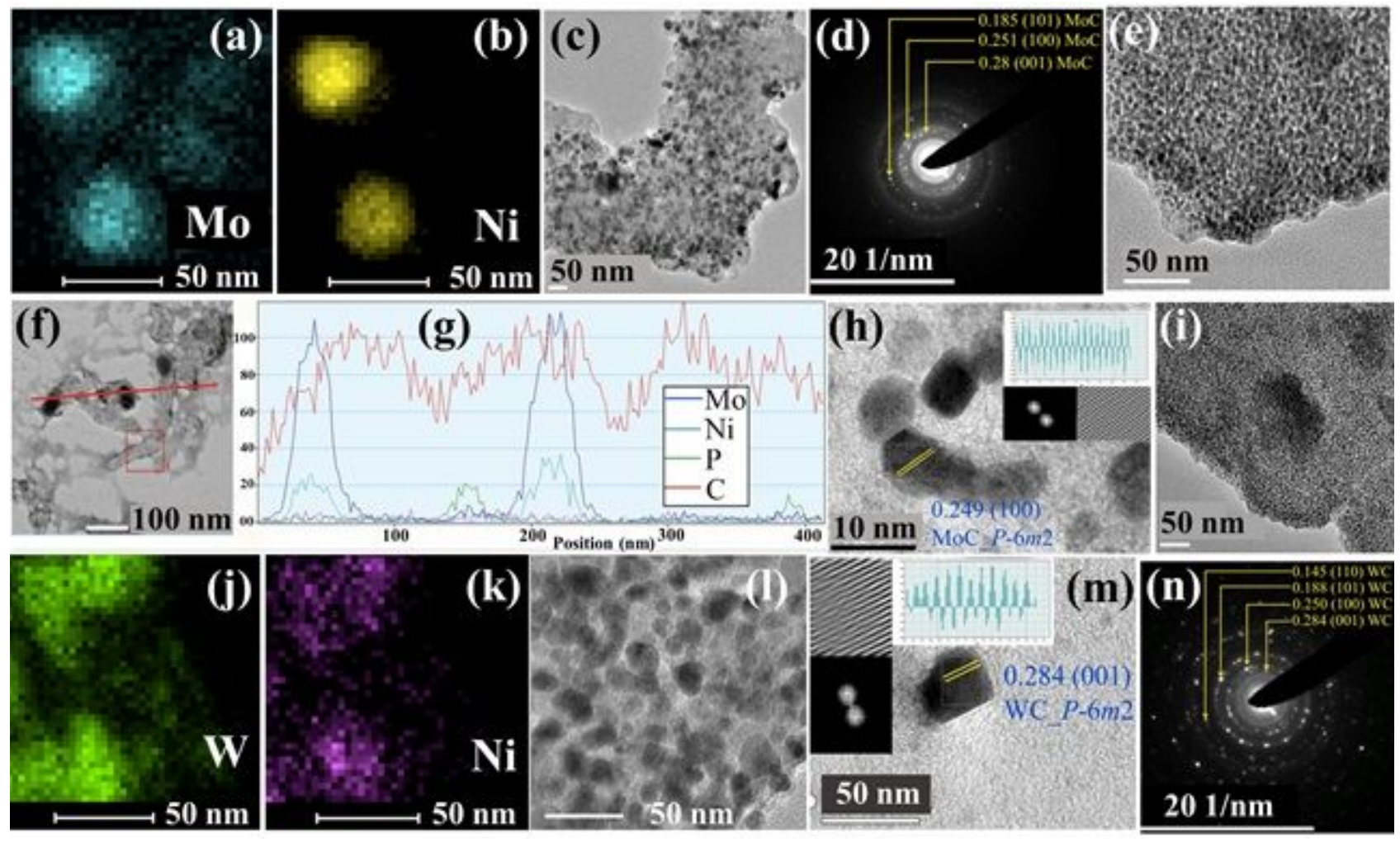

Figure 2

TEM analysis of Ni-WC@NGC \& Ni-MoC@NGC: (a) \& (b) Colour mapping on particles of Ni-MoC@NGC, (c) \& (e) Particle distribution in Ni-MoC@NGC, (d) SAED pattern of Ni-MoC@NGC showing presence of corresponding lattice planes, (f) \& (g) Line scanning EDS spectra of Ni-MoC@NGC, (h) HR-TEM image NiMoC@NGC particle showng the d-spacing corresponding to 100 plane, (i) \& (l) Particle distribution in NiWC@NGC, (j) \& (k) Colour mapping on particles of Ni-WC@NGC, (m) HR-TEM image Ni-WC@NGC particle showng the d-spacing corresponding to 001 plane and (n) SAED pattern of Ni-WC@NGC showing presence of corresponding lattice planes. 
(a)

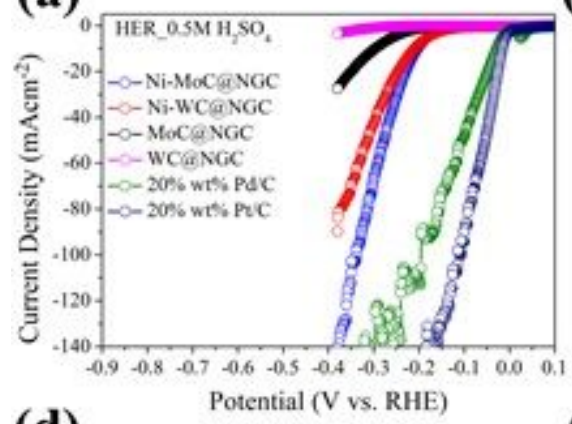

(d)

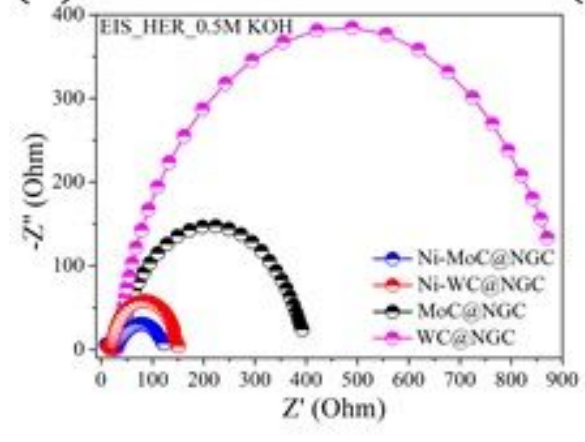

(b)

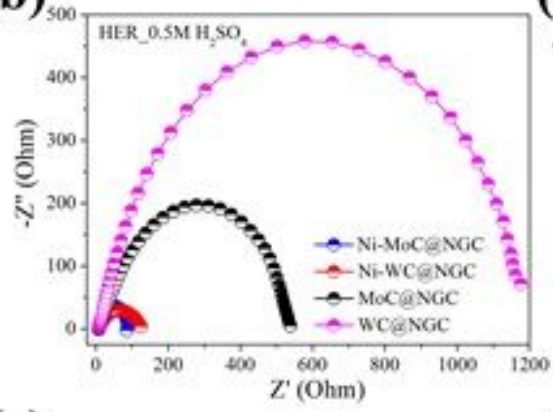

(e)

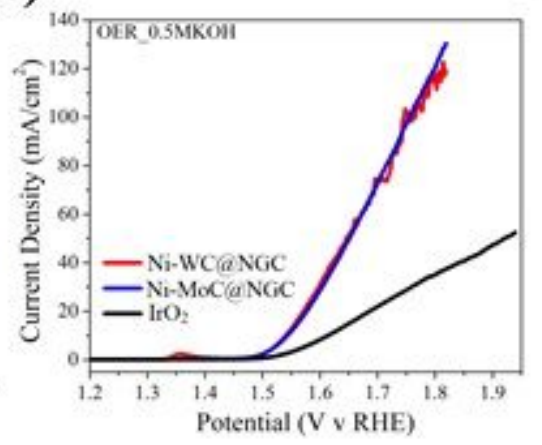

(c)

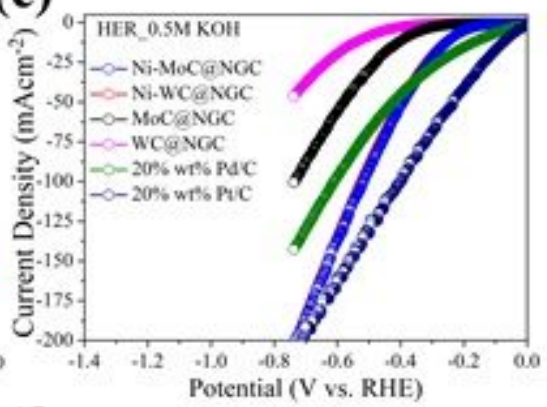

(f)

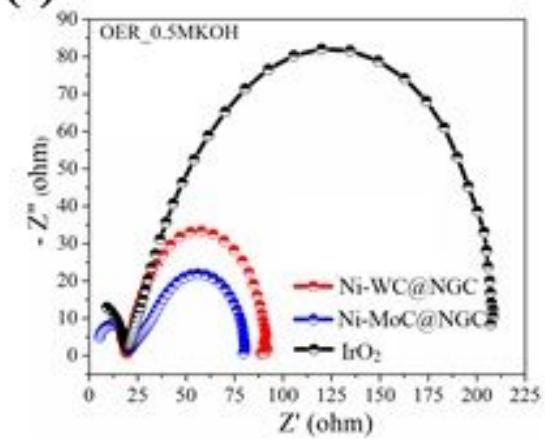

Figure 3

(a) Comparative polarization curves of Ni-WC@NGC \& Ni-MoC@NGC for HER in 0.5M H2SO4 under a scan rate of $5 \mathrm{mV} / \mathrm{s}$ using SCE as the reference and Pt coil as the counter electrode. (b) Electrochemical Impedence Spetra (EIS) of Ni-WC@NGC, Ni-MoC@NGC and undoped catalysts showing RCT during HER in 0.5M H2SO4. (c) Comparative polarization curves of Ni-WC@NGC \& Ni-MoC@NGC with Pd/C \& Pt/C for HER in 0.5M KOH. (d) EIS spectra of Ni-WC@NGC, Ni-MoC@NGC and undoped catalysts showing RCT during HER in $0.5 \mathrm{M} \mathrm{KOH}$. (e) Comparative polarization curves of Ni-WC@NGC, Ni-MoC@NGC and IrO2 for electrochemical OER in $0.5 \mathrm{M} \mathrm{KOH}$ under a scan rate of $5 \mathrm{mV} / \mathrm{s}$ using $\mathrm{Hg} / \mathrm{HgO}$ as the reference and Pt coil as the counter electrode. (f) EIS spectra of Ni-WC@NGC, Ni-MoC@NGC and IrO2 showing RCT during OER in $0.5 \mathrm{M} \mathrm{KOH}$. 
(a)

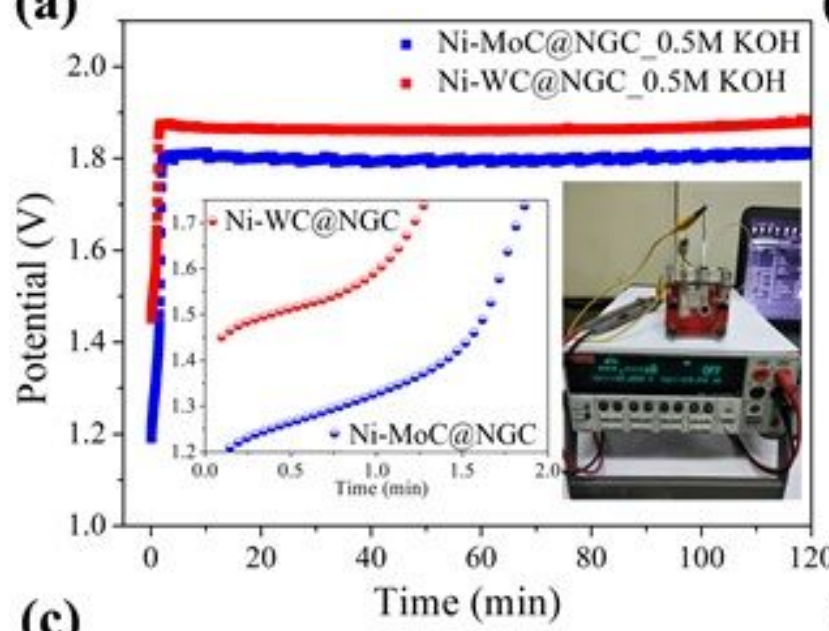

(c)

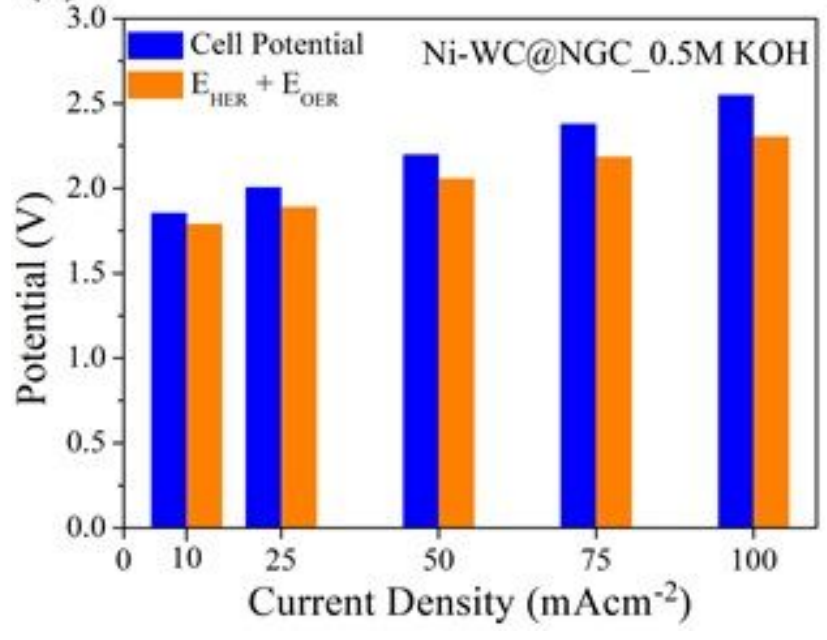

(b)

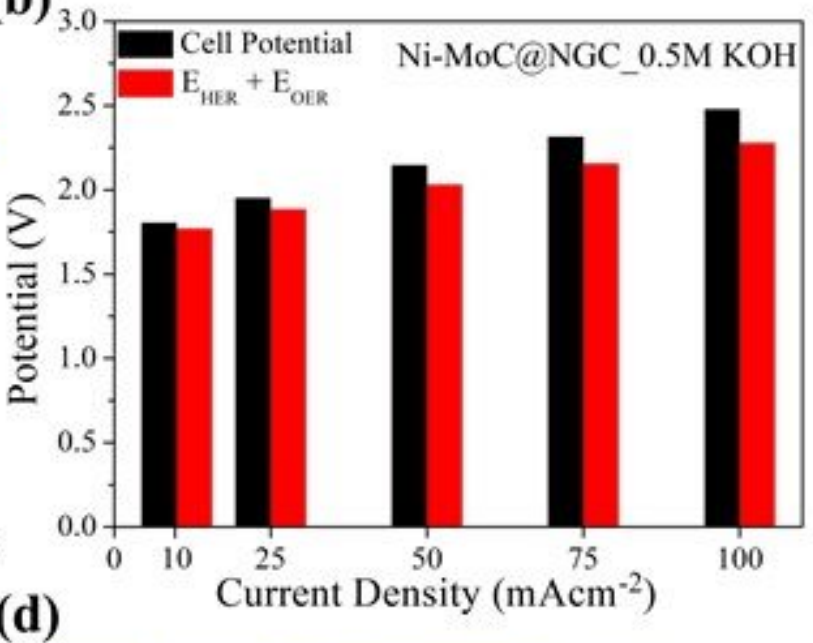

(d)

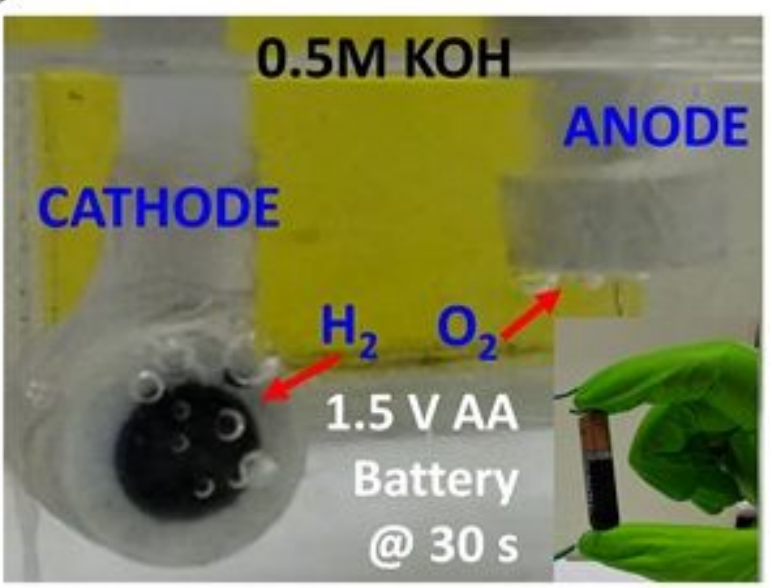

Figure 4

Overall water splitting activity of Ni-WC@NGC \& Ni-MoC@NGC in 0.5 M KOH: (a) Comparative overall water splitting activity and stability of Ni-WC『Ni-WC \& Ni-MoC『Ni-MoC electrolyzers @10mA/cm2 powered by a Keithly Source Meter. The comparison of overall cell potentials obtained experimentally at various current densities under the two electrode setup with the calculated Ecell as the sum of the cathodic and anodic potentials at those current densities during individual HER/OER activity in $0.5 \mathrm{M}$ KOH for (b) Ni-MoC@NGC and (c) Ni-WC@NGC. (d) The evolution of H2 and 02 gas bubbles at the cathode and anode (single chamber cell, two electrode setup) respectively aided by a $1.5 \mathrm{~V}$ AAA commercial battery, after $30 \mathrm{sec}$ of voltage application. 

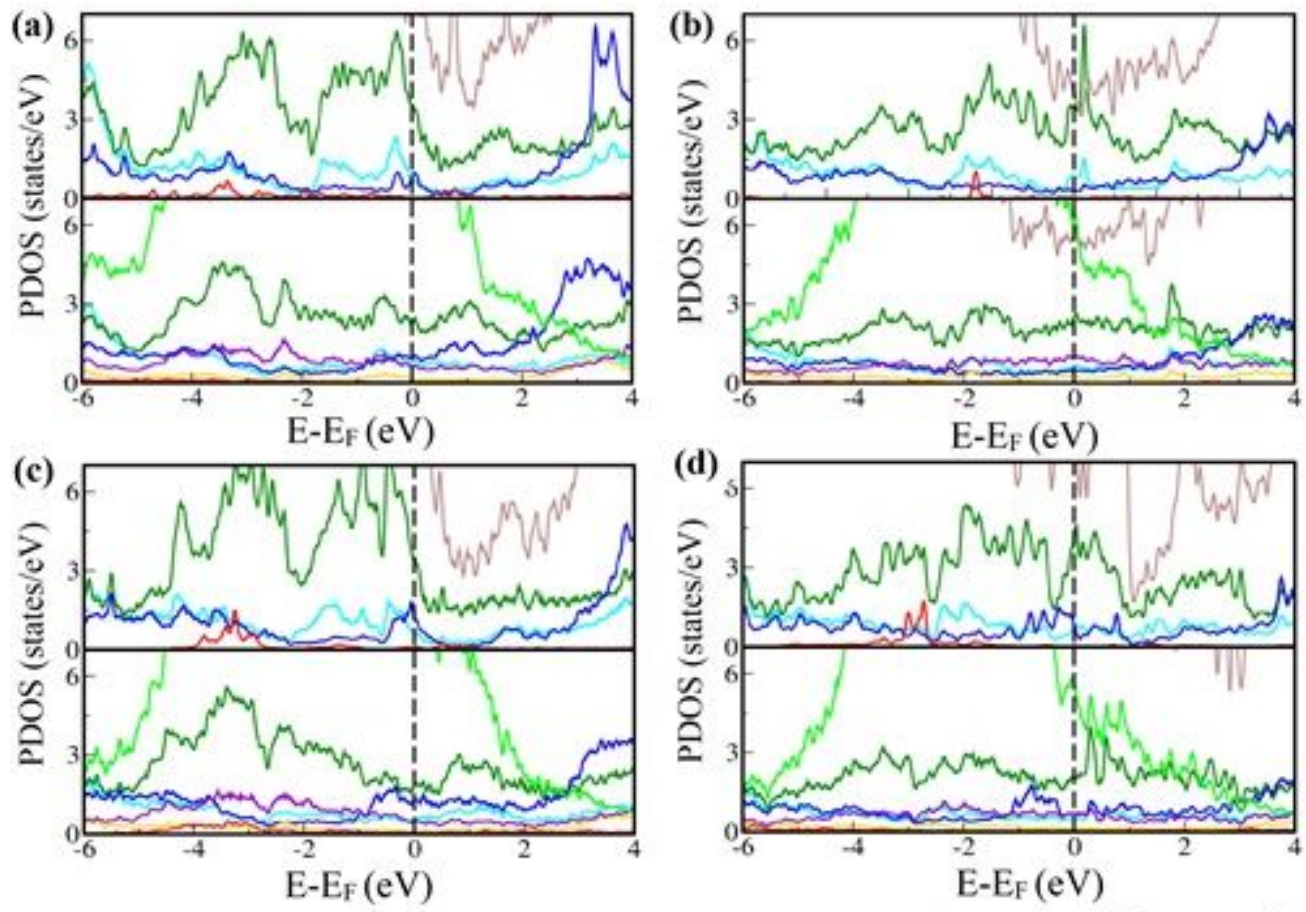

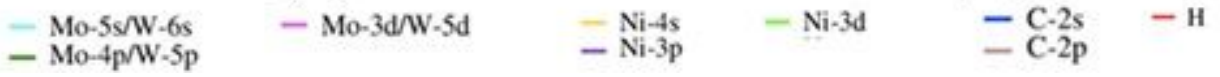

Figure 5

Projected density of states (PDOS) of structures with H-adsorbed on pristine (top) and Ni-Mo/WC(1:5) substituted surfaces (bottom) of (a) MoC (100), (b) MoC (101), (c) WC (100) and (d) WC (101) surfaces. Dashed black lines represent the Fermi level (EF). PDOS (zoomed out) including all the orbitals is shown in Figure S18.

(a)

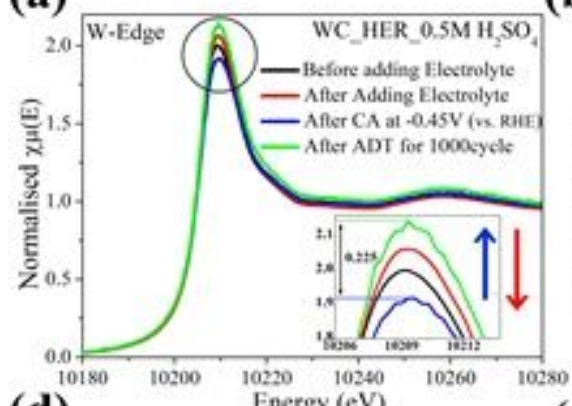

(d)

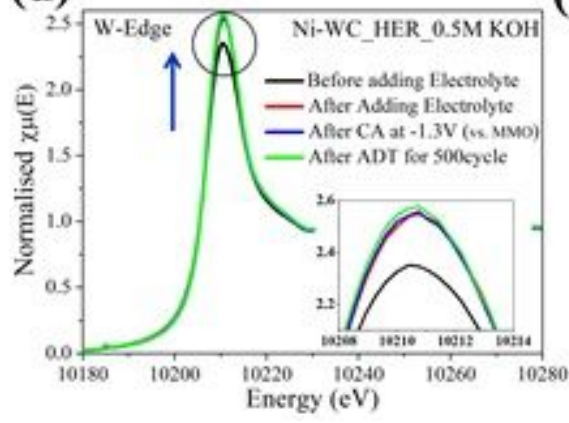

(b)

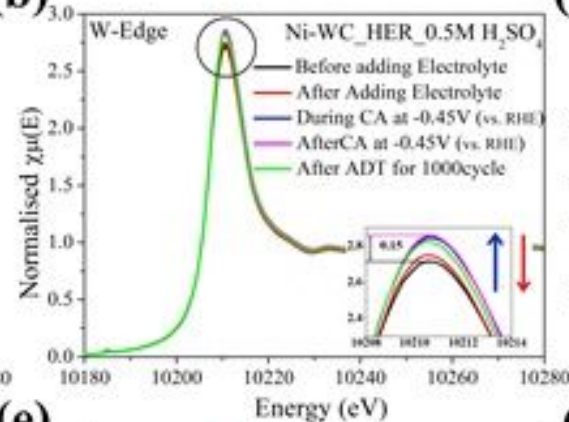

(e)

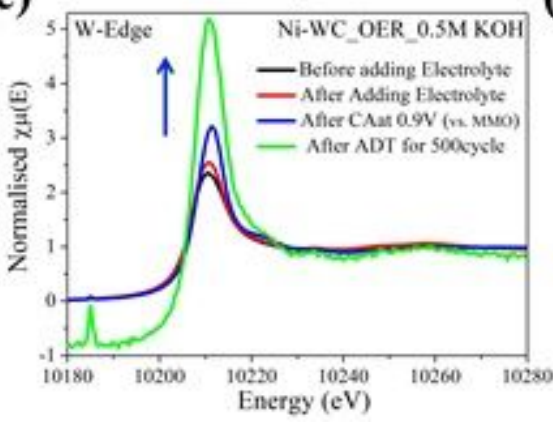

(c)
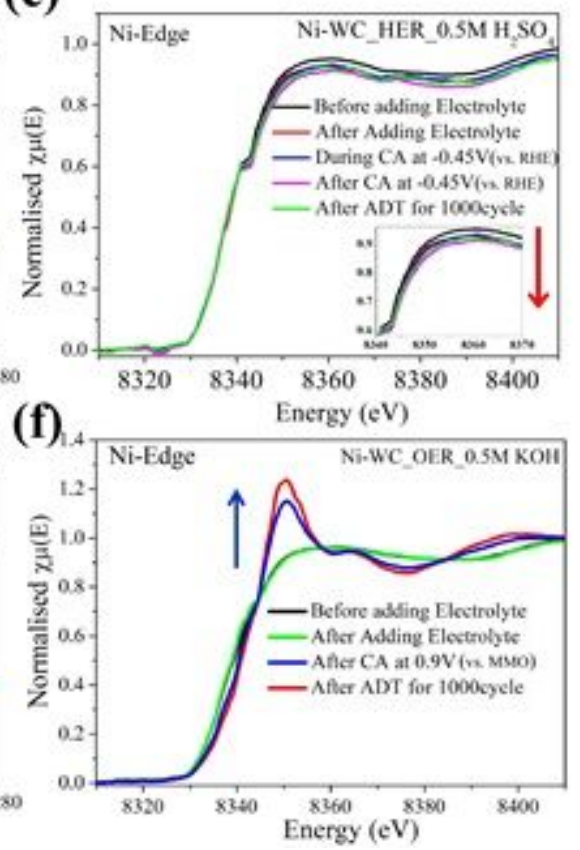

Figure 6 
In-situ XANES studies at (a) W edges of WC@NGC during HER in 0.5M H2SO4, (b) W edges of NiWC@NGC during HER in in 0.5M H2SO4, (c) Ni edges of Ni-WC@NGC during HER in 0.5M H2SO4, (d) W edges of Ni-WC@NGC during HER in 0.5M KOH, (e) W edges of Ni-WC@NGC during OER in 0.5M KOH and (f) Ni edges of Ni-WC@NGC during OER in 0.5M KOH.

\section{Supplementary Files}

This is a list of supplementary files associated with this preprint. Click to download.

- OperandoAnalysisMetalCarbidesWaterSplittingSI.docx

- VideoS1.mp4

- VideoS1.mp4

- VideoS1.mp4

- VideoS2.mp4

- Videos2.mp4

- Videos2.mp4

- OperandoAnalysisMetalCarbidesWaterSplittingSI.docx

- OperandoAnalysisMetalCarbidesWaterSplittingSI.docx

- OperandoAnalysisMetalCarbidesWaterSplittingSI.docx 